Elsevier Editorial System(tm) for Polymer Manuscript Draft

Manuscript Number:

Title: Peculiarities of Photonic Crystal Recording in Functional Polymer Nanocomposites by Multibeam Interference Holography

Article Type: Research Paper

Section/Category: Synthesis and Chemistry of Polymers

Keywords: Photonic crystal, Polymer nanostructures, Multibeam interference holography

Corresponding Author: Dr. Istvan Csarnovics, PhD

Corresponding Author's Institution: Insitute of Physics, University of Debrecen

First Author: Dmitrii Zhuk

Order of Authors: Dmitrii Zhuk; Julia Burunkova; Ihor Denisyuk; Nina Mirosnichenko; Istvan Csarnovics, PhD; Dávid Tóth; Attila Bonyár; Miklós Veres; Sándor Kökényesi

Abstract: Two-dimensional photonic crystal structures (PCS) were fabricated using a one-step recording process, multi-beam interference in smart polymer nanocomposites incorporating SiO2 and Au nanoparticles sensitized to $532 \mathrm{~nm}$ laser radiation. It was shown, that PCS with different geometry can be recorded in thick nanocomposite layers. The typical two dimensional (2D) PCS have square structure with 2-8 $\mu m$ period, being in good agreement with theoretical structures obtained by mathematical modeling of the recorded PCS. The peculiarities of the photo-polymerization of nanocomposites with plasmonic Au nanoparticles were analyzed on the basis of Surface Plasmon Resonance Imaging (SPRi) and Raman spectroscopy investigations and used for the interpretation of the recording process and periodic structure formation. 
Dear Editor Board of Polymer.

As a co-author of the manuscript, which title: Peculiarities of Photonic Crystal Recording in Functional Polymer Nanocomposites by Multibeam Interference Holography

I would like to submit our research work to the Polymer. The manuscript includes original and novel results, which are important in developing new methods of synthesis and optical transparent polymer nanocomposites for surface pattern creation and fabrication of two-dimensional photonic crystal structures. The results are presented in high quality way.

All figure captions have been provided. The manuscript has not been previously published, is not currently submitted for review to any other journal, and will not be submitted elsewhere before a decision is made by this journal. The English of the manuscript was polished. The titles of the tables and figures in the reference list were added.

I would kindly ask you to accept our paper.

Authors:

1. D. I. Zhuk - University ITMO, St. Petersburg, 197101, Russian Federation

2. J. A. Burunkova - University ITMO, St. Petersburg, 197101, Russian Federation

3. I. Yu. Denisyuk - University ITMO, St. Petersburg, 197101, Russian Federation 4. N. P. Mirosnichenko - University ITMO, St. Petersburg, 197101, Russian Federation

5. I. Csarnovics - Institute of Physics, University of Debrecen, Hungary 
6. D. Tóth - Institute of Physics, University of Debrecen, Hungary

7. A. Bonyár - Department of Electronics Technology, Budapest University of

Technology and Economics, Budapest, Hungary

8. M. Veres - Wigner Research Centre for Physics, Hungarian Academy of

Sciences, Budapest, Hungary

9. S. Kokenyesi - Institute of Physics, University of Debrecen, Hungary

On behalf of the authors,

Dr. István Csarnovics

corresponding author

assistant professor 

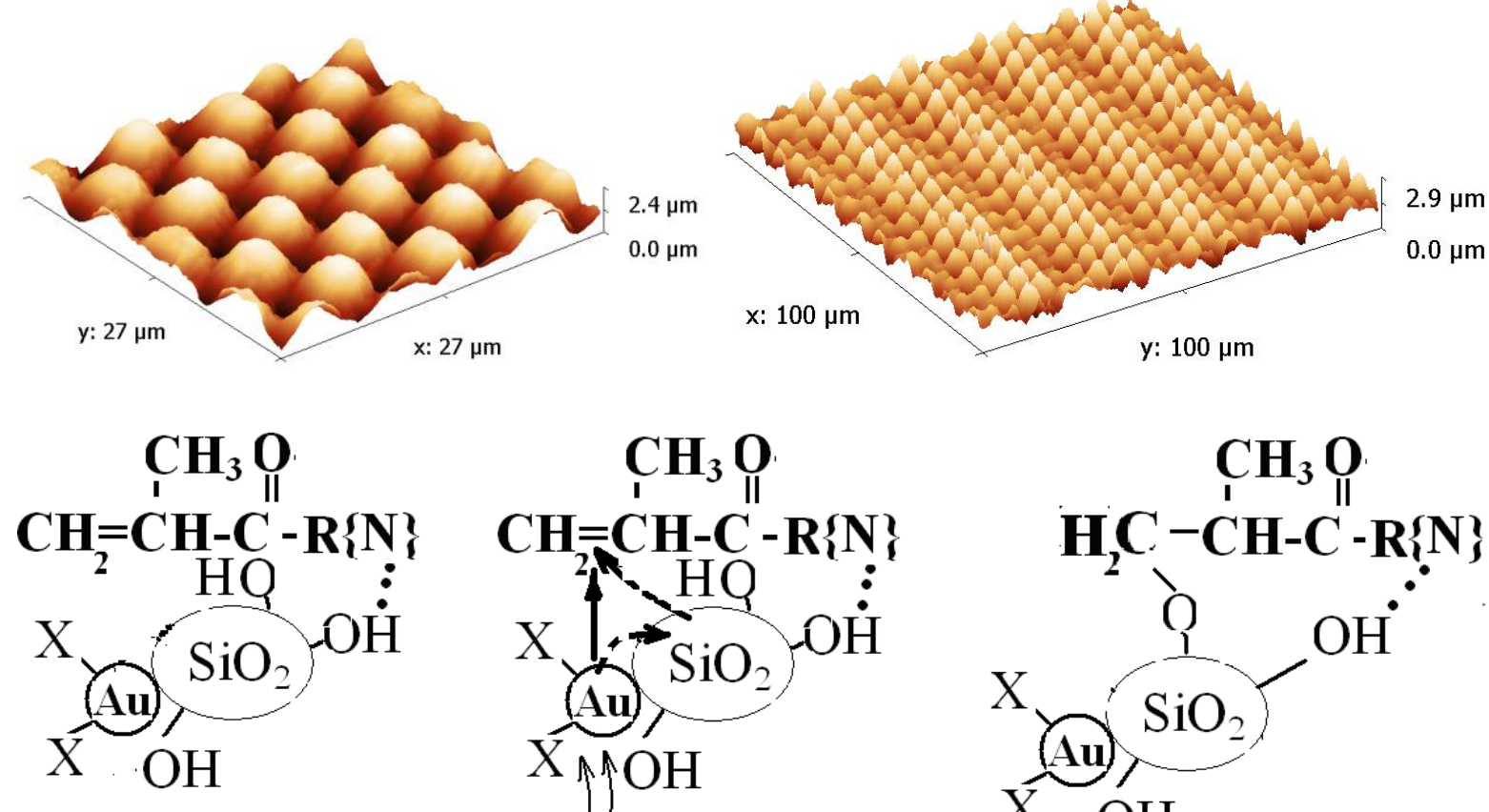

$\longrightarrow \quad 532 \mathrm{~nm}$

$\mathrm{CH}_{3} \mathrm{O}$

$\mathrm{CH}=\mathrm{CH}-\mathrm{C}-\mathrm{R}\{\mathrm{N}\}$

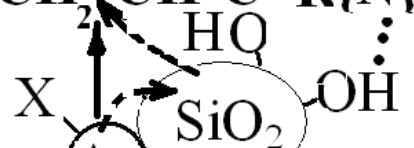

(Au) $\mathrm{SiO}_{2}$

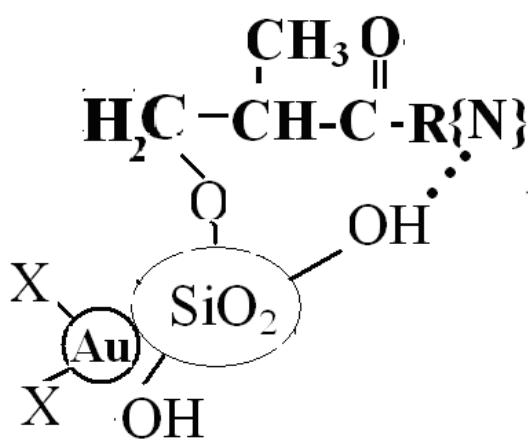




\title{
Peculiarities of Photonic Crystal Recording in Functional Polymer Nanocomposites by Multibeam Interference Holography
}

\author{
D.I. Zhuk ${ }^{1}$, I.A. Burunkova ${ }^{1}$, I.Yu. Denisyuk ${ }^{1}$, N. P. Mirosnichenko ${ }^{1}$, \\ I. Csarnovics ${ }^{2}$, D. Tóth ${ }^{2}$, A. Bonyár ${ }^{3}$, M. Veres ${ }^{4}$, S. Kokenyesi ${ }^{2}$ \\ ${ }^{1}$ University ITMO, St. Petersburg, 197101, Russian Federation \\ ${ }^{2}$ Institute of Physics, University of Debrecen, Hungary \\ ${ }^{3}$ Department of Electronics Technology, Budapest University of Technology and Economics, \\ Budapest, Hungary \\ ${ }^{4}$ Wigner Research Centre for Physics, Hungarian Academy of Sciences, Budapest, Hungary
}

\begin{abstract}
Two-dimensional photonic crystal structures (PCS) were fabricated using a one-step recording process, multi-beam interference in smart polymer nanocomposites incorporating $\mathrm{SiO}_{2}$ and Au nanoparticles sensitized to $532 \mathrm{~nm}$ laser radiation. It was shown, that PCS with different geometry can be recorded in thick nanocomposite layers. The typical two dimensional (2D) PCS have square structure with $2-8 \mu \mathrm{m}$ period, being in good agreement with theoretical structures obtained by mathematical modeling of the recorded PCS. The peculiarities of the photopolymerization of nanocomposites with plasmonic $\mathrm{Au}$ nanoparticles were analyzed on the basis of Surface Plasmon Resonance Imaging (SPRi) and Raman spectroscopy investigations and used for the interpretation of the recording process and periodic structure formation.
\end{abstract}

\section{Introduction}

During the last decades photonic crystals attract essential basic scientific and increasing application oriented interests for the development of integrated optics, sensors, infocommunication technologies [1-3]. A wide number of materials and proper fabrication methods and processes were developed: lithography, e-beam or laser-beam recording, holography [4-7]. Lithography can be used for photonic crystals' (PC) fabrication in basic semiconductor materials like silicon [4]. Such structures contain elements with dimensions in the micrometer range. Nanoimprint method was proposed [7] for the fabrication of one and two dimensional PCS in polymethyl-methacrylate, structures with $800 \mathrm{~nm}$ period were obtained this way. Using special multi-faced prism, which splits the laser beam into four beams and converges them afterwards on a light-sensitive material, authors of [8] recorded PC with forbidden gap at $1400 \mathrm{~nm}$. In [8] 3D PC was fabricated by the multi-beam holographic lithography in SU-8 photoresist using single optical element, which formed beams' convergence at selected necessary angles.

The most of the well-known fabrication methods have a number of technological shortcomings. For example, the application of the multi-face prism or optical element, which converges light beams do not allow an in situ variation of interference patterns. Application of holographic lithography for PCS fabrication makes the additional step of selective etching necessary, with all possible shortcomings of this process. This method makes it possible to fabricate a large photonic element by continuous, step by step incorporation of separate elements. Lithography method, together with copying and multiplexing allows us to obtain PCS down to sub-micrometer dimensions in semiconductors and polymers. Electron-beam lithography makes it possible to produce elements with much higher resolution, but the low throughput restricts its application in a large scale production. Application of lithography with copying, imprint technology provides possibility for serial production of simple surface structures. Here problems can appear with selective etching of prototype elements.

The range of known materials for PCS is rather wide: thin films of noble metals, silicon, different semiconductors and polymers. Hybrid materials for PCS are perspective, especially 
with combination of photonic and plasmonic properties; they are so called hybrid photonicplasmonic heterocrystals [9].

In the present paper periodical structures in acrylate- $\mathrm{SiO}_{2}$ nanocomposites are formed by holographic method of multi-beam interference of coherent light in a system of four beams. In this method the formation of the recording beams provides a possibility of creation of periodical structures with different forms and dimensions without changes of optical elements. Photonic structures are formed in special acrylate-urethane nanocomposites, which contain silica nanoparticles $\left(\mathrm{SiO}_{2} \mathrm{NP}\right)$ and were developed and used in our laboratories. Some of them contain gold nanoparticles (AuNP) besides the $\mathrm{SiO}_{2} \mathrm{NP}$, which can essentially influence on the polymerization process and on the formation of periodical structures [10]. It is essential, that no additional treatments of the material after the recording are necessary.

The goal of the present work was the development and application of the above mentioned multi-beam interference method for one step formation of photonic structures in functional acrylate nanocomposites, which are sensitized to green laser illumination and possess plasmonic effects due to the presence of gold nanoparticles.

\section{Experimental}

\subsection{Sample preparation.}

Monomer compositions were used for holographic recording of periodical structures. These materials are mixtures of urethane - acrylate monomers with $\mathrm{SiO}_{2} \mathrm{NP}$ and photo-initiator, which is sensitive in the green region of optical spectrum. This spectral region was selected specially, since in our experiments we use monomer compositions with AuNP, which possess plasmon resonance and absorption just in the green spectral region.

The technology of nanocomposite preparation consists of following steps:

- $\mathrm{SiO}_{2} \mathrm{NP}$ (7 $\mathrm{nm}$ in diameter) are added by small portions into the mixture of diurethane dimethacrylate (436909 Aldrich, UDMA) and isodecilacrylate (408956 ALDRICH, IDA),

- Adding toluene to the mixture to decrease the viscosity of it.

- After mixing the composition, the necessary amount of AuNP in toluene is added.

- Further the initiator of photo-polymerization is added (Irgacure 784, bis-(5-2,4ciklopentadien-1-il) bis-[2,6-difluor-3-(1H-pirolle-1-il) phenyl ] titanium.

- Afterwards the toluene is evaporated at $35^{\circ} \mathrm{C}$ till the constant weight. The composition is stored at $25^{\circ} \mathrm{C}$.

The composition of the prepared nanocomposites is shown in Table 1.

\begin{tabular}{|c|c|c|c|c|}
\hline № & Monomers & $\begin{array}{c}\mathrm{SiO}_{2} \mathrm{NPs} \\
\mathrm{wt} \%\end{array}$ & $\begin{array}{c}\mathrm{Au} \mathrm{NPs} \\
\mathrm{wt} \%\end{array}$ & $\begin{array}{c}\text { Irgacure 784 } \\
\text { wt\% }\end{array}$ \\
\hline 64a & UDMA/IDA=1/2 & 10 & - & 0,5 \\
\hline 64Au1 & UDMA/IDA=1/2 & 10 & 0.08 & 0,5 \\
\hline 64Au2 & UDMA/IDA=1/2 & 10 & 0.15 & 0,5 \\
\hline
\end{tabular}

Table 1. Composition of the prepared nanocomposites.

\subsection{Experimental setup for recording of PCS}

The general scheme of the experimental setup is presented in Fig.1. 


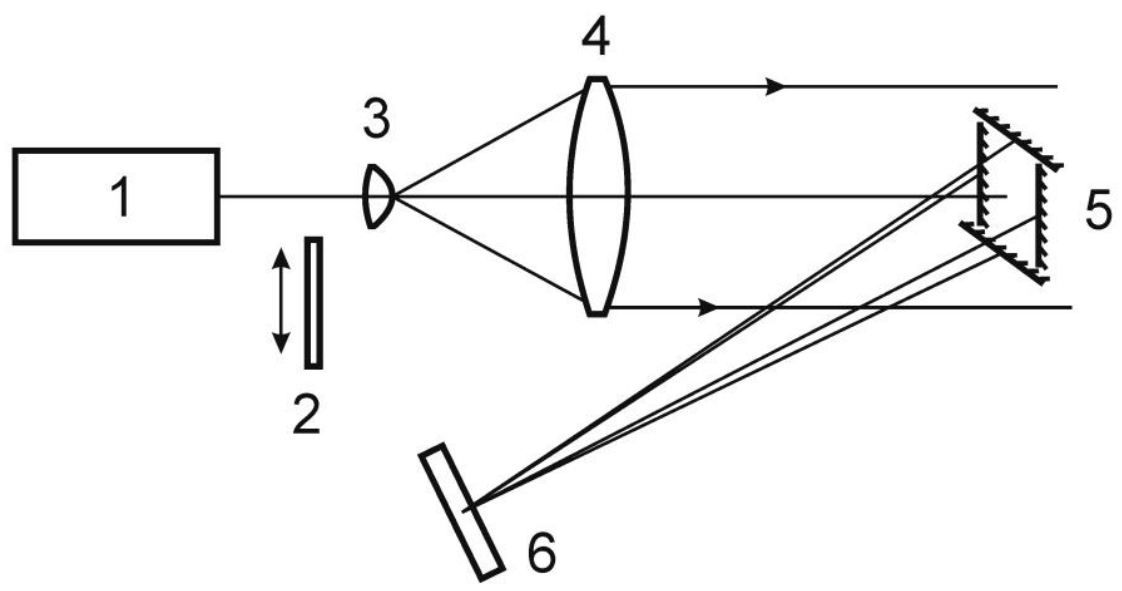

Fig. 1. Scheme of PC recording. 1 - DPSS laser $(\lambda=532 \mathrm{~nm})$;

2 - optical filter; 3,4 - telescope with wide aperture, 5 - four-mirror system, 6 - the sample with monomer nanocomposite layer.

The beam of light with $532 \mathrm{~nm}$ wavelength and $100 \mathrm{~mW}$ power from single-mode DPSS laser is widened by telescope system with lenses ( 3 and on Fig.1) to the diameter, which is enough for uniform illumination of the four-mirror system (5 on Fig. 1). The diameter of the illuminated field in this system of mirrors was near $80 \mathrm{~mm}$.

Each of four mirrors is attached to the separate micrometer stage, which in turn are attached to the separate common stage with micro-positioners in two directions. This way four independently operated beams are available in the given optical system, and their diameters are determined by the dimensions of mirrors, i.e. the mirrors serve as limiting diaphragms at the same time. Further, the beams are settled to the position 6 , where the nanocomposite sample is placed. The intensity of beams can be regulated by light filters ( 2 on Fig.1). The adjustment of four beams for obtaining interference picture was done by the method of continuous approximations with aim to select the minimum difference between optical paths of beams from the given laser. At each step of approximation the interference picture was monitored through an optical microscope, located at the sample place (6 on Fig.1). The adjustment process was finished if a good quality periodical structure was observed.

The recording zone, where the four beams intercept, was usually about 10x10 mm, although the size could be increased or decreased as well. It is necessary to notice that this system of mirrors allows high accuracy regulation of beam convergence and overlap different illuminated zones. Different periodical structures can be produced this way.

The following PCS were created:

PCS 1 - monomer of $64 \mathrm{a}, 3$ minutes recording.

PCS 2 - monomer of 64a, 3 minutes recording, and one beam was shifted for $2 \mathrm{~mm}$ in vertical direction relative to other three beams in comparison to PCS 1.

PCS 3 - monomer of 64Au1, 3 minutes recording, the same as for PCS 1.

\subsection{Theory of interference holography through multi-beam interference}

The electric field for each $p=1,2,3,4$ beam can be determined by the following equation of a plane wave with wavelength $\lambda$, unit vector along the wave vector $\boldsymbol{\varepsilon}^{(p)}=\left(\sin \left(\alpha_{p}\right) \cos \left(\phi_{p}\right), \sin \left(\alpha_{p}\right) \sin \left(\phi_{p}\right), \cos \left(\phi_{p}\right)\right)$, with azimuth angles $\alpha_{p}$ and polar angles $\phi_{p}$ : 


$$
\boldsymbol{E}^{(p)}(\boldsymbol{r}, t)=\boldsymbol{A}^{(p)} \cdot \exp \left(i \frac{2 \pi}{\lambda}\left(c t-\left(\boldsymbol{\varepsilon}^{(p)}, \boldsymbol{r}\right)\right)\right)
$$

Angles are determined relative to the vector perpendicular to the surface of layer (sample 6 in Fig.1). The $z$ axis is perpendicular to the sample surface, $x, y$ axes are in the sample plain. The electric field vector is denoted as $\boldsymbol{e}^{(p)}$. Then,

$$
\boldsymbol{A}^{(p)}=A^{(p)} \boldsymbol{e}^{(p)} .
$$

Polar angles of $\boldsymbol{e}^{(p)}$ vectors are equal to zero (since the electric field vectors are directed along the $x$ axis). The azimuth angles of the $\boldsymbol{e}^{(p)}$ vectors are denoted as $\beta_{p}$. At such conditions, when the electric field vector vibrates perpendicularly to the direction of propagation, the $\beta_{p}$ angles can be described by the next equation:

$$
\beta_{p}=\beta_{p}\left(\alpha_{p}, \phi_{p}\right)=\pi-\operatorname{arcctg}\left(\operatorname{tg}\left(\alpha_{p}\right) \cos \left(\phi_{p}\right)\right) .
$$

Intensity distribution in the interference picture obtained from four beams can be described by the following equation:

$$
I(x, y)=\frac{c}{8 \pi}\left|\sum_{p=1}^{4} \boldsymbol{E}^{(p)}(\boldsymbol{r}, t)\right|^{2}
$$

Let the beams be characterized by equal amplitudes $A^{(1)}=A^{(2)}=A^{(3)}=A^{(4)}=A$, and equal azimuth angles $\alpha^{(1)}=\alpha^{(2)}=\alpha^{(3)}=\alpha^{(4)}=\alpha$.

An example of modeled structure is shown in Fig. 2d. Here the intensity distribution of fourbeam interference at the surface of the sample layer is given at the conditions: azimuth angle $\alpha=0.065$ radian, polar angles of wave vectors in radians are: $\phi_{1}=0, \phi_{2}=\frac{\pi}{2}, \phi_{3}=\pi, \phi_{4}=\frac{3 \pi}{2}$. The scale on $x, y$ coordinates is selected in $\mu m$, the wavelength equals $0.532 \mu \mathrm{m}$. Intensity $I(X, Y)$ is in arbitrary units on $z$ axis. The intensity distribution at the surface of sample at four- beam interference with azimuth angle $\alpha=0.065$ radian and polar angles $\phi_{1}=0, \phi_{3}=\pi, \phi_{4}=\frac{3 \pi}{2}$ is presented in Fig. $3 \mathrm{~d}$. The second beam is turned in such a way, that the polar angle equals $\phi_{2}=\frac{\pi}{2}+0.35$ radian.

\subsection{Investigation of the prepared PCS.}

The 2D and 3D images of the created PCS, their cross-section, period and average modulation depth were measured by atomic force microscope (AFM - Veeco diCaliber). The optical spectra of the pure samples and their PCS were measured with Shimadzu 4016 UV/VIS spectrometer. Optical microphotography was taken by "Olympus" microscope. The structure of the created PCS was measured by a Renishaw 1000 micro-Raman spectrometer using the 488 
$\mathrm{nm}$ line of an Ar-ion laser as excitation source. The baseline-corrected spectra were normalized to their most intense peak at $1450 \mathrm{~cm}^{-1}$.

The photo-polymerization process of the created samples was investigated by measuring the refractive index change during irradiation. The refractive index change caused by a green light emitting diode (LED) (operating wavelength $522 \mathrm{~nm}$ ) illumination was measured by Surface Plasmon Resonance imaging (SPRi). The light beam of the sources was defocused, so the intensity of illumination on the sample was $2 \mathrm{~mW} / \mathrm{cm}^{2}$. For the measurements a custom-built SPRi instrument [11] was used which utilizes Kretschmann optical configuration with a $680 \mathrm{~nm}$ superluminescent light source and a $1 \mathrm{MP}$ CCD camera with $25^{\circ}$ range of incident angle. Realtime changes in the refractive index of the media were monitored during the whole duration of the illumination. SPRi results (kinetics) are always given as absolute changes of the refractive index of the nanocomposites with time as it was shown earlier [11]..

\section{Results and discussion of holographic recording of PCS in acrylate nanocomposites}

In situ formation of periodical structures (photonic crystals) was performed by the interference field in light sensitive composites, which consist of organic monomers and inorganic nanoparticles. The photo-polymerization and diffusion processes result in the periodical distribution of the nanoparticles in the matrix material. This mechanism of diffraction grating recording in polymer composites was analyzed earlier, see for example [12].

The sample with nanocomposite layer, placed in the recording position (6 on Fig. 1) was exposed for $3 \mathrm{~min}$ by the preliminary adjusted beams, which gave one form of periodical structure. This exposure time was enough for the photo-polymerization and lateral diffusion of monomers and nanoparticles. This process results in a hard polymer film with an inscripted pattern of the multi-beam interference. The image, with the distribution of the elements depends on the convergence angles of the recording beams, as it is shown on Fig. 2a, which is recorded in UDMA/IDA monomer mixture and demonstrated the characteristic feature of a two-dimensional periodical structure (PCS 1). Fig. $2 b$ demonstrates the diffraction of $\lambda=635 \mathrm{~nm}$ light on this structure. The calculated and real structures are presented in Fig. $2 \mathrm{~d}$ and $2 \mathrm{c}$ respectively, while an AFM image and its cross-sectional profile are given in Fig 2.e and 2f. The period of the PCS 1 is $7.88 \mu \mathrm{m}$, the average modulation depth equals to $2 \mu \mathrm{m}$. 


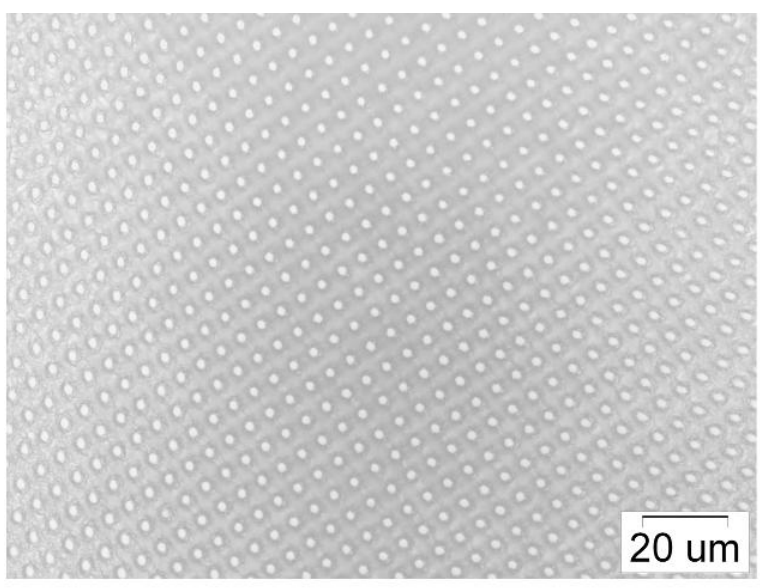

a
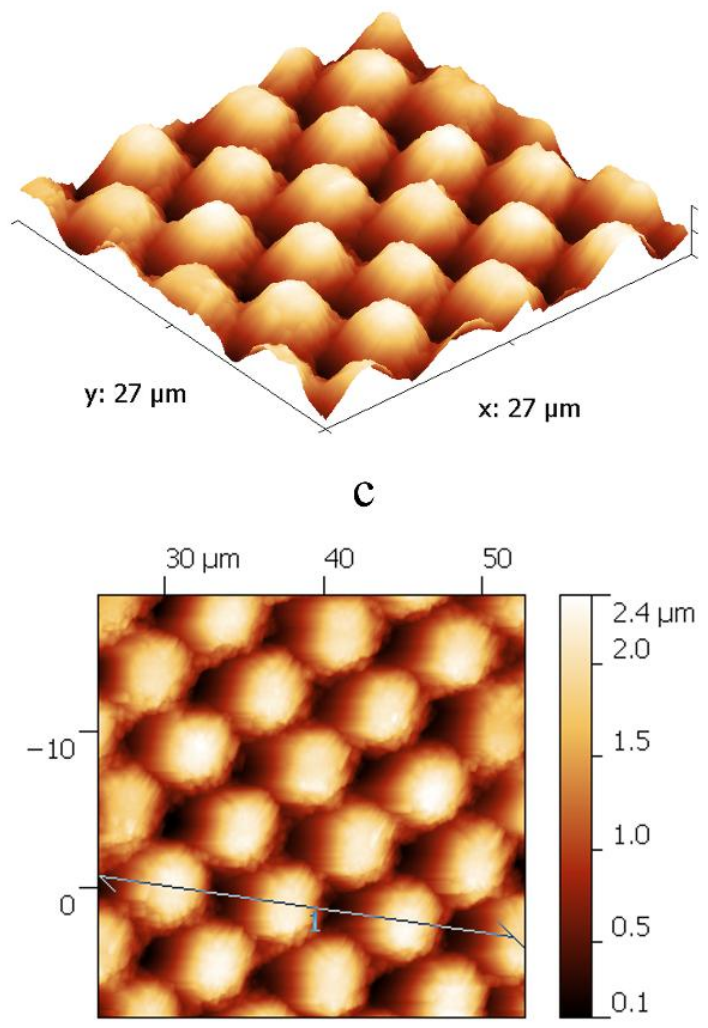

$\mathrm{e}$

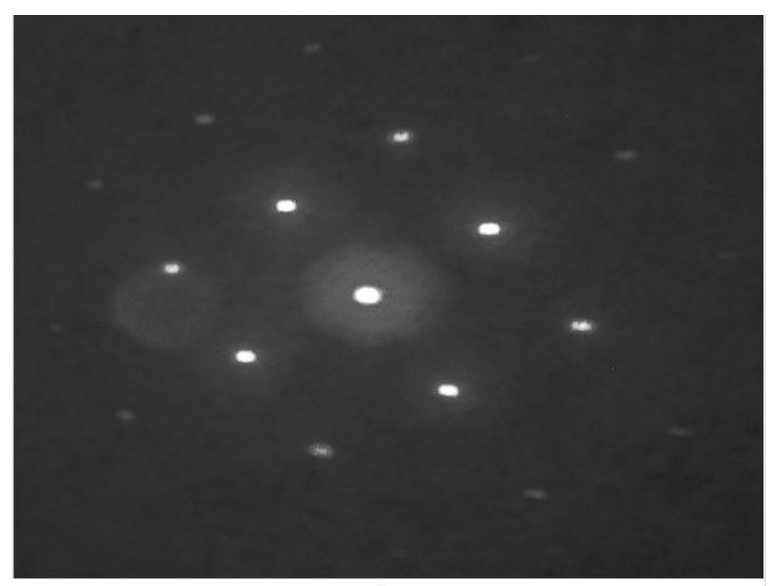

b
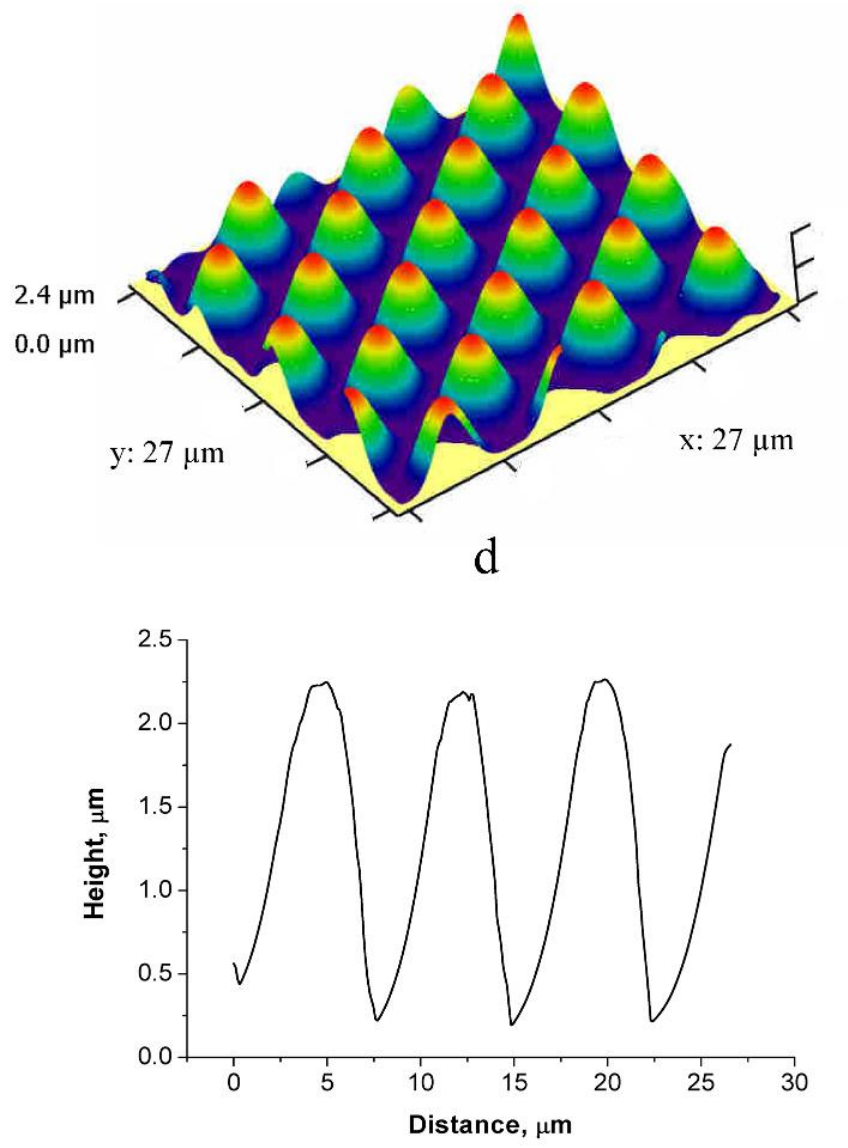

f

Fig. 2. Microstructure of the PCS 1:

a - optical microphotography,

b - light diffraction on the 2D PC,

c - 3D AFM picture 2D PCS,

d - calculated distribution of light intensity at four-beam interference with symmetrical adjusting of beams,

e - AFM image of the created PCS,

$\mathrm{f}$ - surface profile according to the marked line in $2 \mathrm{e}$. 
PC with different geometrical structures can be formed by this method in the developed nanocomposite material, at different arrangements of the recording beams. The adjustment was always done through an optical microscope. For example, if one beam is extracted from the interference, the resulting structure can be essentially changed. One possible result of recording at other converging angles is demonstrated in Fig. 3. Here one beam was shifted for $2 \mathrm{~mm}$ in vertical direction relative to the other three beams.

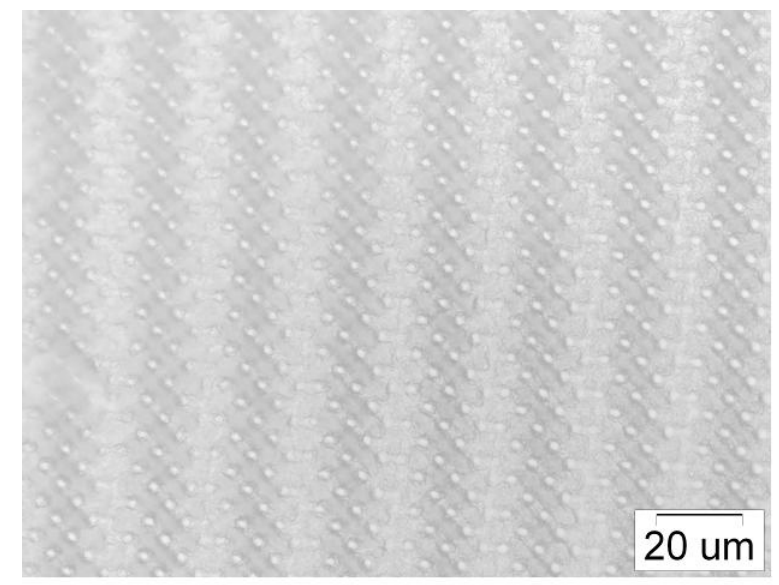

a
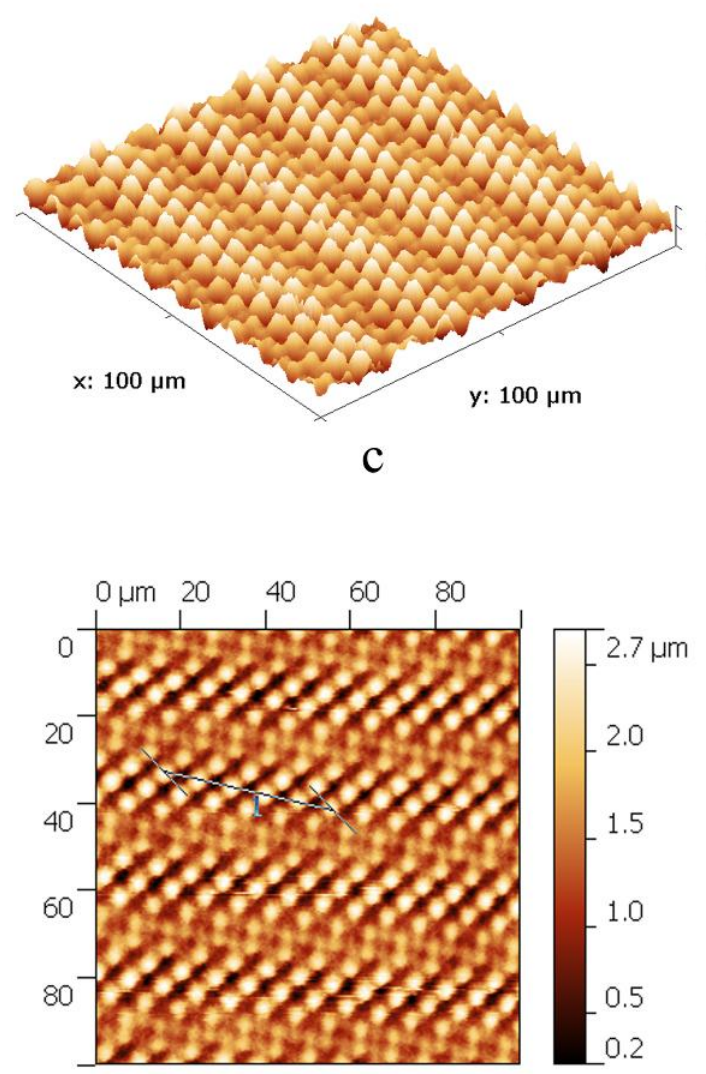

$\mathrm{e}$

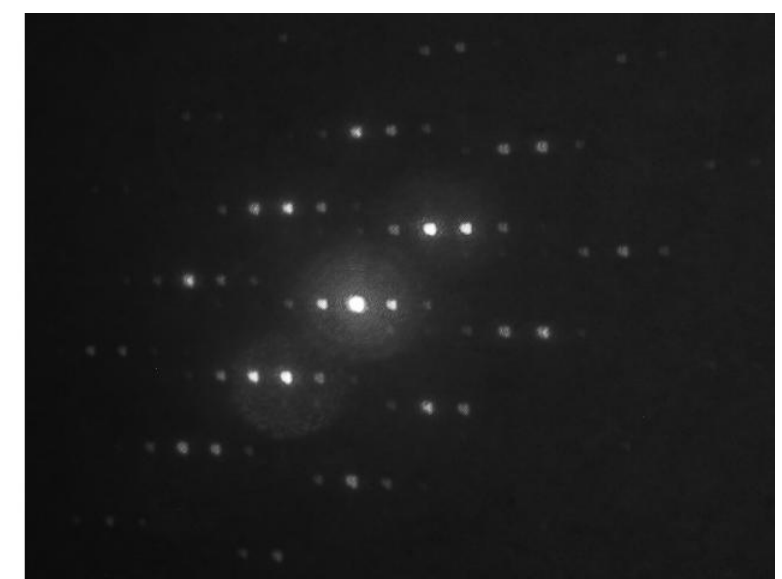

b
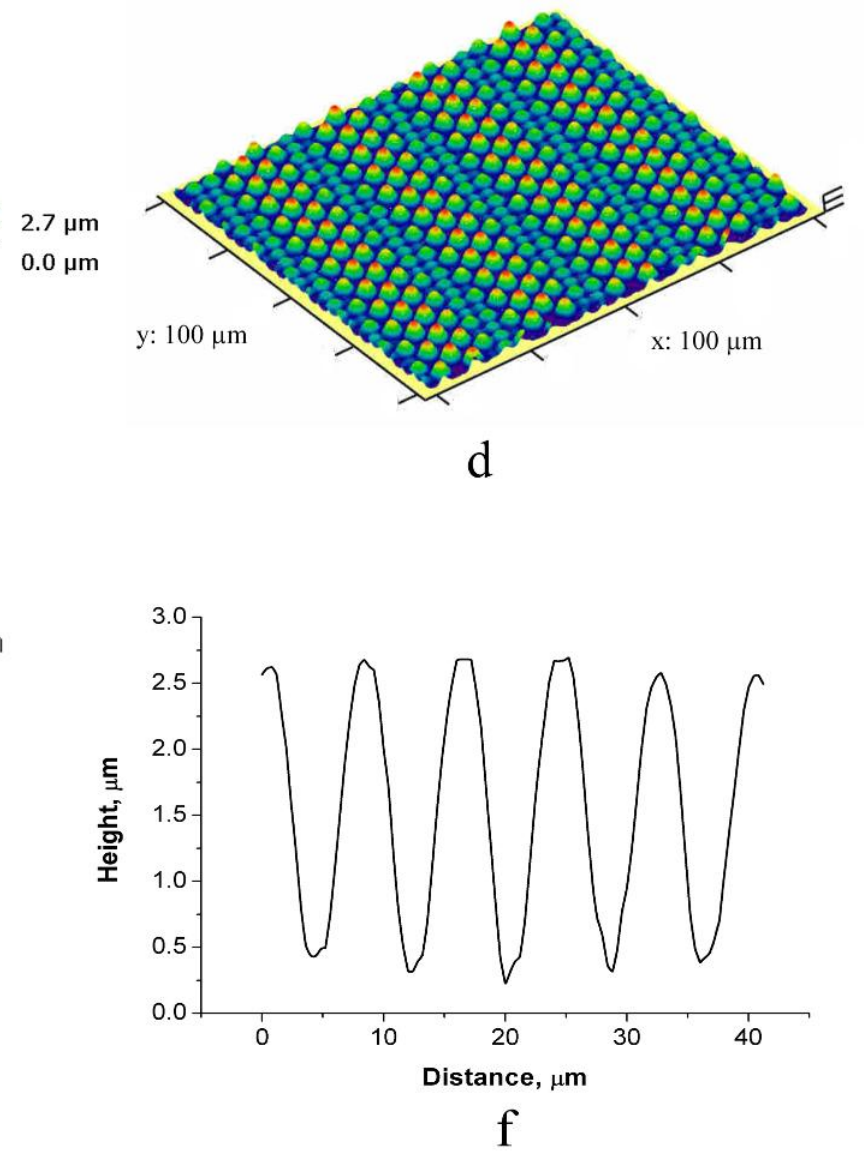

Fig 3. . Microstructure of the PCS 2: a - optical microphotography, b - light diffraction on the 2D PC, 
c - 3D AFM picture of 2D PCS,

$\mathrm{d}$ - calculated distribution of light intensity at four-beam interference with symmetrical

adjusting of beams,

e - AFM image of the created PCS,

$\mathrm{f}$ - surface profile according to the marked line in $3 \mathrm{e}$.

It was established that the simple shift $(2 \mathrm{~mm})$ of one beam relative to the other three results other PC with shifted structures. Two parameters of PCS 2 had been changed in comparison with PCS 1: the average modulation depth from 2.0 to $2.4 \mu \mathrm{m}$, and the period from $7.88 \mu \mathrm{m}$ to $8.2 \mu \mathrm{m}$. It is important, that the phase modulation at readout of this structure consists of not only a simple surface component, but the volume one as well, which appears due to the volume redistribution of nanoparticles. The flat surface the diffraction is the same (compare Fig. $2 \mathrm{~b}$ and 4$)$. So, we have a bulk PCS.

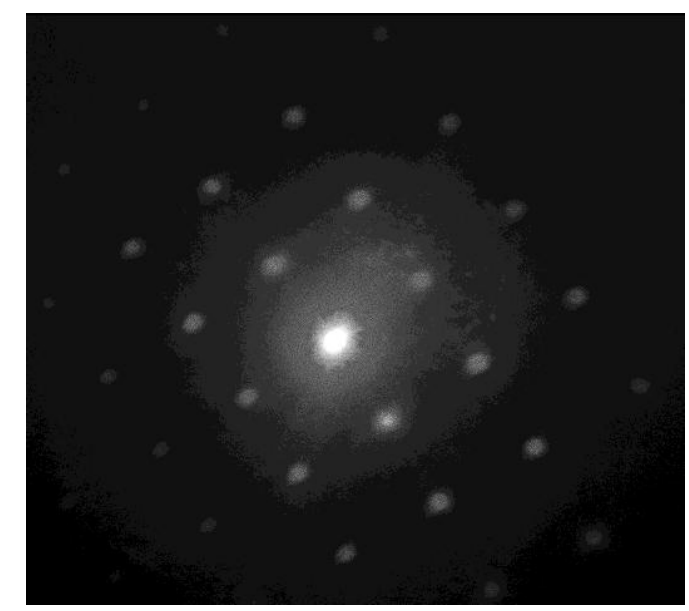

Fig. 4. Diffraction pattern obtained on 2D PCS with additional surface polymer cover.

The topology of the recorded surface structures correlates well with the calculations: comparison of $2 c-2 d$ and $3 c-3 d$ pictures demonstrates it. So the mathematical model describes the interference patterns well at the given experimental conditions. This gives us the possibility to create optimal structures with the desired parameters.

Furthermore, we have investigated the PCS recording in functionally upgraded lightsensitive nanocomposite material towards the possibility of recording at the presence of plasmon fields, generated by excited AuNPs. Here the peculiarities of polymerization processes, which were investigated earlier for gold-containing nanocomposites at blue-and UV-light excitation, can be modified. PCS 1 was recorded on sample 64Au, which contained $0.15 \mathrm{wt} \%$ of AuNPs. The created structures were examined via AFM. It was established, that at the given conditions of recording and parameters of the green-light sensitive nanocomposite the efficiency of direct surface deformations, i.e. the PCS relief depth decreases in comparison with the PCS 1 structure. The same effect was observed at recording simple one-dimensional PCS, i.e. diffraction grating in this nanocomposite material with and without AuNPs and the diffraction efficiency of the resulting Bragg gratings was lower in the composites with AuNPs.

Besides that, the plasmon resonance absorption is present in this elements, as well as in the initial nanocomposite (see Fig. 5), but redistributed according to the redistributed AuNPs in the recorded relief (see Fig.6). The possible distribution of the nanoparticles between hillocks and valleys of recording pattern were investigated by micro-Raman spectroscopy (see Fig. 6). The $1450 \mathrm{~cm}^{-1}$ and $1641 \mathrm{~cm}^{-1}$ peak on Fig. 6 corresponds to the alkyl group and to the carboncarbon double bonds, consequently. The first peak does not change during recording, so it could be used as reference to control the change of other functional group. It could be shown on Fig. 6c that the relative intensity peaks of the $\mathrm{C}=\mathrm{C}$ bonds are higher for valley in comparison to hillock. 
It could be concluded, that hillock is a light field of the recording pattern (it was more irradiated), while the valley is dark one (it was less irradiated). The photo-polymerization rate is higher at hillock, therefore, nanoparticles should diffuse to the darker areas of irradiation valleys. The strong line at $530 \mathrm{~cm}^{-1}$ is corresponds to the symmetrical vibrations of $\mathrm{Si}-\mathrm{O}-\mathrm{Si}$ tetrahedras in siloxanes, while peaks at $610 \mathrm{~cm}^{-1}$ and $750 \mathrm{~cm}^{-1}$ are correspond to vibrations of the C-S, (dodecanethiol group of AuNPs) (see Fig 6c). As it is evident from Fig. 6c the intensity of the bands connected with nanoparticles and their concentration are higher in the valley in comparison to hillock (Fig. 6c). So as a result of pattering the nanoparticles were redistributed. The PCS with AuNPs could be further used for creation of sensor elements upgraded with plasmon enhanced functions.

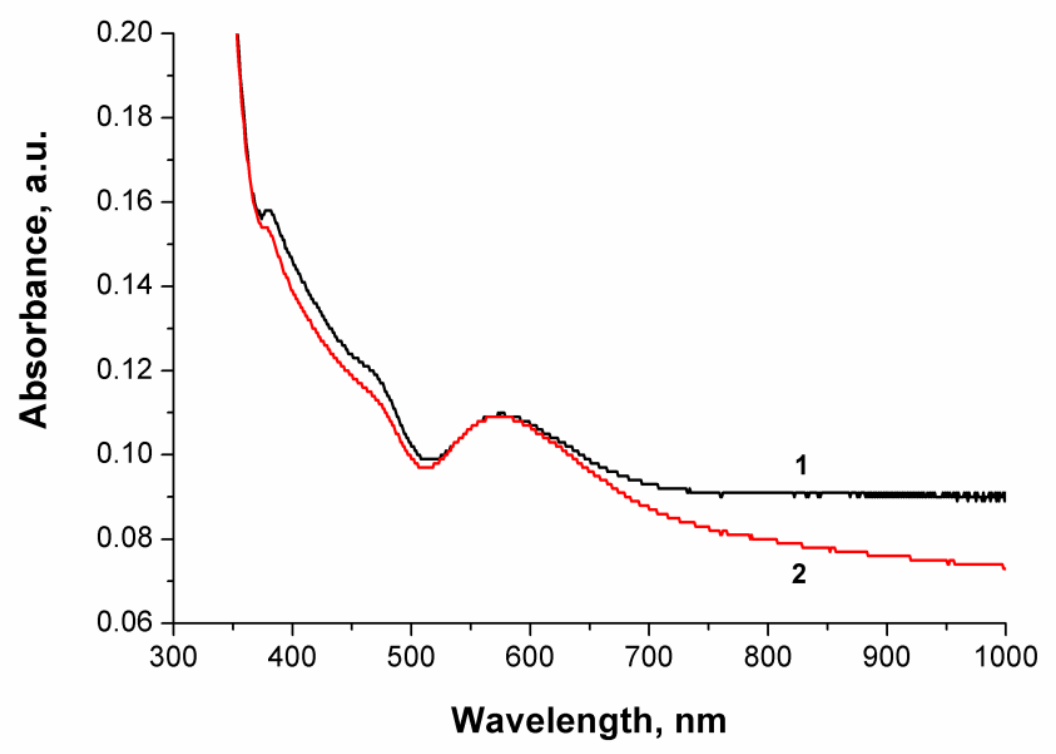

Fig. 5. Optical absorbance spectra of the nanocomposite layer with AuNPs (1) and of the 2D PCS 1, recorded in this layer (2).
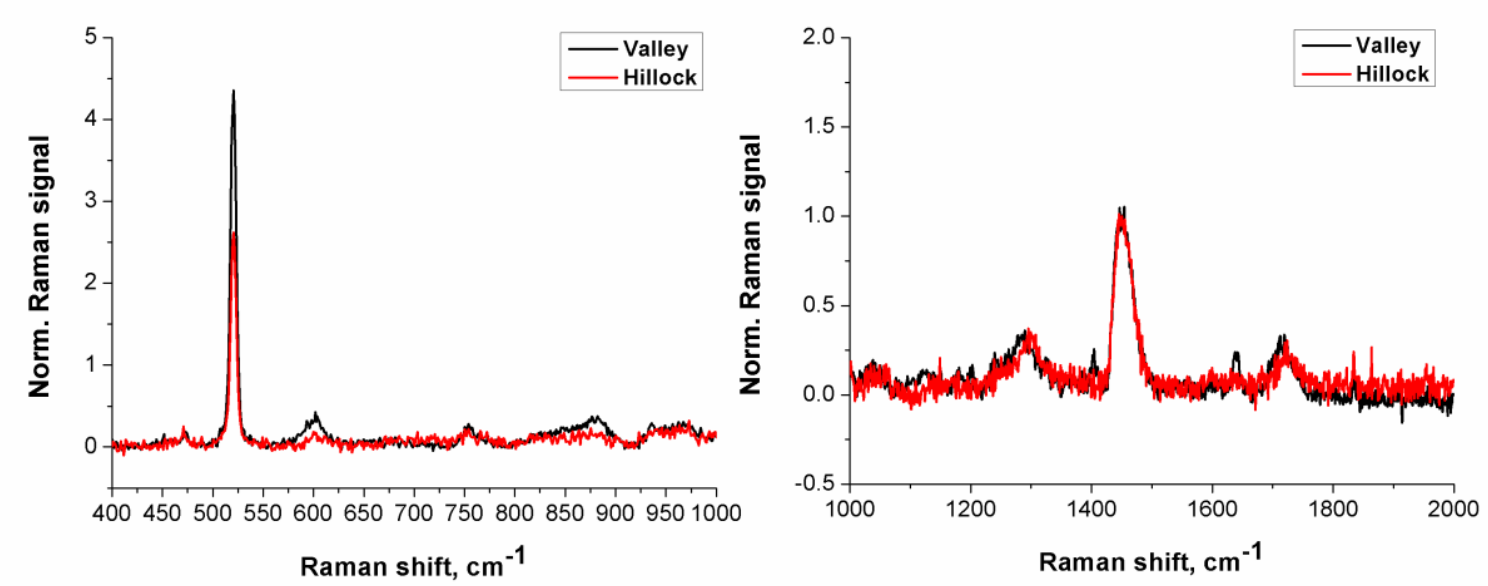

Fig. 6. MicroRaman mapping of $\mathrm{SiO}_{2} \mathrm{NP}$ and AuNPs concentration distribution along the grating vector in a simple PCS - diffraction grating recorded on 64Au2.

The results that were presented, an important question may arise regarding the role of the AuNPs in the process of photo-polymerization. It has a direct chemical influence on the polymerization-diffusion processes at PCS recordings and it is an important issue in the above 
described fabrication technology. This issue can be even more important if we intend the further functionalization of PCS by other molecules (e.g. rare earth materials for luminescence properties), creation of special sensor elements, etc. Additional experiments were done to explain the role of AuNPs in the investigated recording processes.

First, surface plasmon resonance imaging was used for in situ monitoring of the photopolymerization process of the nanocomposite, based on the measurement of the refractive index change under the influence of $522 \mathrm{~nm}$ irradiation. Results are presented in Fig. 7.

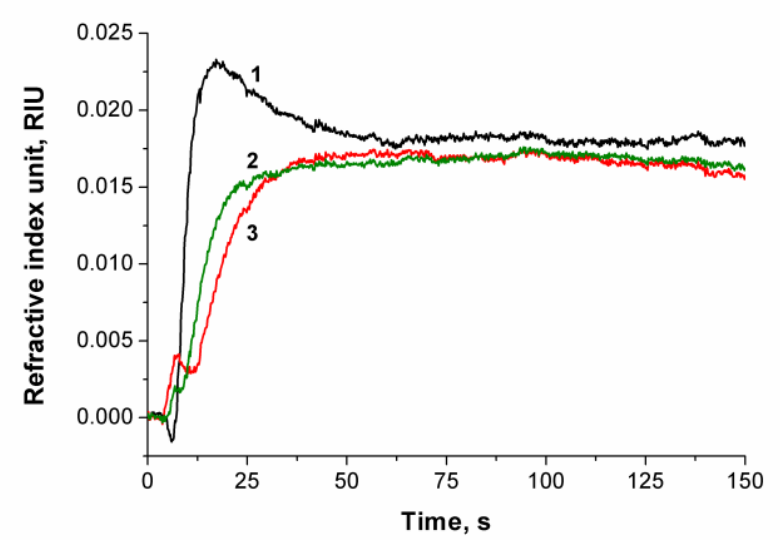

Fig. 7. SPRi measurement results regarding the refractive index changes of the different nanocomposites under the influence of irradiation: 1 -64a, 2 -64Au1, 3-64Au2.

The essential difference between the process in compositions without and with AuNPs is the presence of initial maximum of refractive index change in Au-free nanocomposite. This can be explained in the following way. It is known, that the photo-polymerization at the initial stage of the process takes place at the surface of $\mathrm{SiO}_{2} \mathrm{NP}$ [13]. As a result of this process a denser polymer structure with higher refractive index is formed in comparison with the structure in the $\mathrm{SiO}_{2}$-free volume. From the certain moment of irradiation the share of volume polymerization increases in comparison with polymerization at the $\mathrm{SiO}_{2}$ surface, the density and the refractive index become averaged and results in saturation plateau.

This effect can be explained as the following. The nanocomposite consists of two monomers, which differ in photo-polymerization rates and refractive indexes: UDMA possesses higher polymerization rate and higher refractive index in comparison with IDA. In our case, the polymer with higher refractive index is polymerized at first on the surface of $\mathrm{SiO}_{2} \mathrm{NP}$. The denser structure is formed and the maximum is observed on the time-refractive index dependence (see Fig.7, curve 1). Further, the volume polymerization prevails, the share of component with lower refractive index increases and saturation is observed (see Fig.7, curve 1). Also it was established from SPRi measurements for our nanocomposites that the introduction of AuNPs reduces the rate and degree of polymerization (see Fig 7, curve 2). The maximum at the initial stage of photo-polymerization is absent. The concentration increase of AuNP results decrease of the refractive index change rate, i.e. in the decrease of the polymerization rate.

The mechanism of this process, which also influences the fabrication of PCS, can be explained in more details on the basis of microRaman spectroscopy investigations. Examples of these data are presented in Fig.8. 

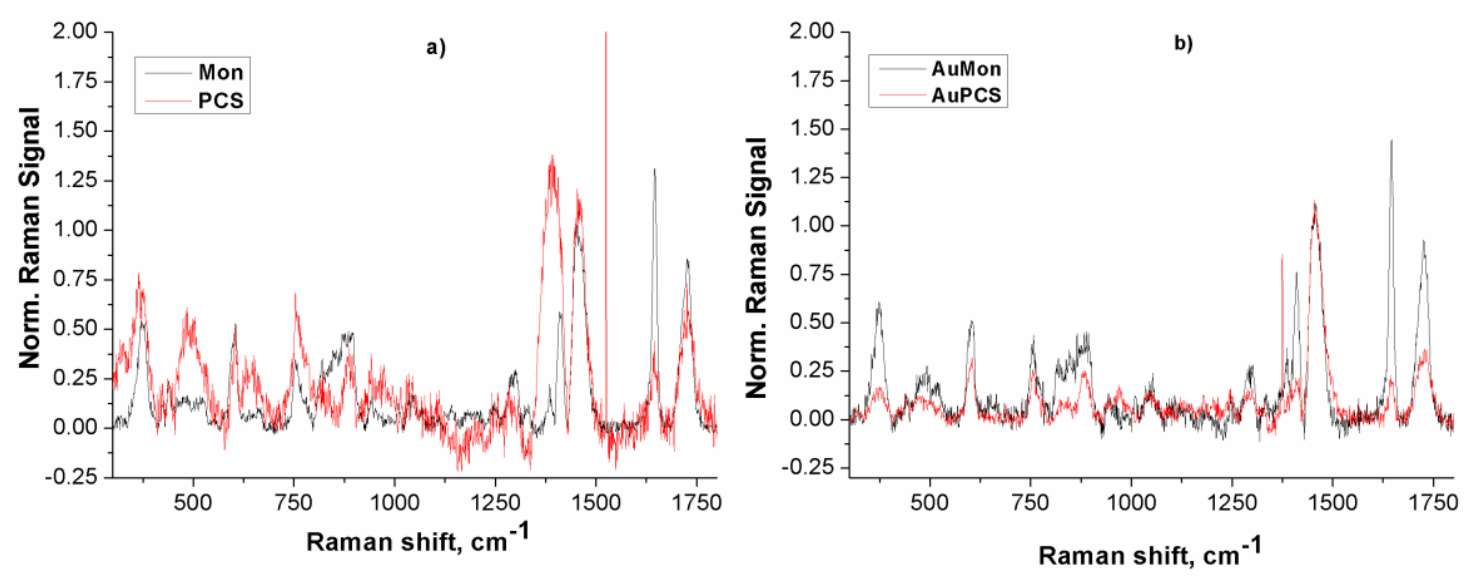

Fig. 8. Raman spectra of monomer $(1,3)$ and cured nanocomposites $(2,4)$ : a)64a - without AuNP, b)64Au2 - with AuNP.

Analyzing the data presented in Fig. 8 for monomer and polymerized samples for nanocomposite with and without AuNPs the vanishing of the

$1410 \mathrm{~cm}^{-1}$ band and the decrease of the $380 \mathrm{~cm}^{-1}$ band can be concluded for $\mathrm{Au}$ containing nanocomposite. The $1410 \mathrm{~cm}^{-1}$ band results from the stretch vibrations of $-\mathrm{OH}$ groups, which are located at the surface of $\mathrm{SiO}_{2}$ nanoparticles. The $380 \mathrm{~cm}^{-1}$ band corresponds to the deformations of the urethane groups [14]. Comparison of the Raman spectra of monomer and polymer - AuNP nanocomposites shows, that under the influence of $522 \mathrm{~nm}$ irradiation the concentration of $\mathrm{SiO}_{2}$ surface-connected $-\mathrm{OH}$ groups and the mobility of urethane framework decrease. All of these can be connected to the polymerization at the surface of $\mathrm{SiO}_{2} \mathrm{NP}$.

Probably, in these processes the AuNPs promote photo-polymerization at the surface of $\mathrm{SiO}_{2} \mathrm{NP}$ under excitation with $522 \mathrm{~nm}$ light source. The possible explanation consists in the transfer of plasmon energy from the excited AuNPs directly to the double bond of monomer or thru the excitation of $\mathrm{SiO}_{2} \mathrm{NPs}$ which further initialize radical polymerization at the $\mathrm{SiO}_{2} \mathrm{NP}$ surface (the last was mentioned as also possible in polymerization processes [13]). The possible route of such process is presented in Fig. 9 .
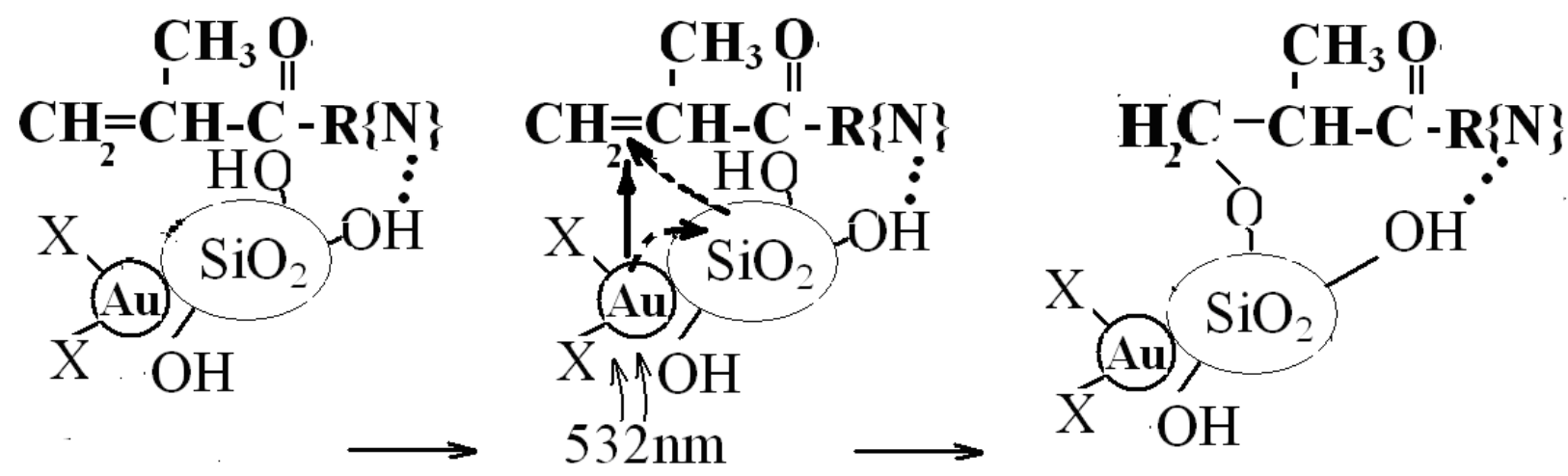

Fig. 9. Polymerization at the surface of $\mathrm{SiO}_{2} \mathrm{NP}$ in the presence of AuNP.

Some decrease of the final refractive index in the composites with AuNPs could be related either to the decrease of the conversion degree or to the increase of the low refractive index share in the average refractive index of the mixed components. It can be seen from the Raman spectra, that the band at $1640 \mathrm{~cm}^{-1}$ almost disappears in both nanocomposites. Obviously, the mechanism presented above gives possibility to increase the share of IDA polymerization in 
the total process due to the IDA polymerization at the $\mathrm{SiO}_{2}$ surface. At the same time the increase of the IDA share in the total amount of polymerized material leads to the decrease of the final refractive index in the polymerized nanocomposite.

As a result of photo-polymerization at the surface of $\mathrm{SiO}_{2} \mathrm{NP}$, their dimensions increase, what in turn decreases their mobility and limits migration to the less illuminated regions of the interference picture when recording it. So the concentration of $\mathrm{SiO}_{2} \mathrm{NP}$ should be smaller in these regions in comparison with recording without AuNPs. This way the presence of AuNPs during the photo-polymerization at the surface of $\mathrm{SiO}_{2} \mathrm{NPs}$ results in the decrease of the refractive indexes of the illuminated and non-illuminated regions, i.e. phase modulation depth. The concentration of $\mathrm{SiO}_{2} \mathrm{NPs}$ in the bright, illuminated regions will be higher in the case of $\mathrm{Au}-$ containing nanocomposite in comparison with the nanocomposite without it. As a result, the height of the bumps and the diffraction efficiency of the recorded surface gratings will decrease.

It can be summarized on the basis of processes described above, that the proposed mechanism of AuNPs influence on the photo-polymerization explains the observed effects, which accompany and affect the holographic recording of PCS in the given acrylate nanocomposites.

\section{Conclusions}

Polymer nanocomposites based on acrylates and silica, as well as gold nanoparticles were fabricated with a special route of direct mixing of preformed nanoparticles to the monomer composition. The introduction of Au NPs was important for the forthcoming development of smart sensing, photonic elements with plasmon effects.

2D PCS were recorded with $532 \mathrm{~nm}$ laser light in this material by the method of fourbeam interference. The recording process in $60 \mu \mathrm{m}$ thick layer was direct, single step and resulted spatially distributed polymerization combined with diffusion and geometrical volume change that served phase modulated structure.

The possibility of different PCS formation by the same optical scheme, with modulation periods in 2-8 $\mu \mathrm{m}$ range was shown. Mathematical model of the optimal optical recording conditions was developed, which correlates well with experimental results.

The mechanism of polymerization processes during the recording was analyzed using the results of additional Raman spectroscopy and SPR measurements in these nanocomposites. In our case the introduction of Au NPs slowed down the rate of the refractive index change and reduced the surface relief depth in the recorded PCS. In this case the role of the localized plasmons generated at $532 \mathrm{~nm}$ in AuNPs consists in the enhancement of polymerization at the silica surfaces, where AuNPs are located as well, and increased yield of nanoparticles with higher diameter and lower diffusion coefficients. The latter slowed down the redistribution of nanoparticles, their diffusion from illuminated to the dark regions of the projected interference pattern. Despite the somewhat less geometrical modulation in the presence of AuNPs, this method is important for the further development of smart photonic elements with plasmonic effects.

\section{Acknowledgements}

The support of Rosobrazovanie grant № 3.432.2014/K "Investigation of methods for three-dimensional grating formation by photo-induced redistribution and fixation of gold nanoparticles in photopolymers" is acknowledged. Attila Bonyár is grateful for the János Bolyai Scholarship.

\section{References}


[1]. J. D. Joannopoulos, S. G. Johnson, J. N. Winn, Robert D. Meade, Photonic Crystals: Molding the Flow of Ligh, Second Edition, Princeton University Press, 2008.

[2]. Y. Shen, C. W. Hsu, Y. X. Yeng, J. D. Joannopoulos, M. Soljačić, Broadband angular selectivity of light at the nanoscale: Progress, applications, and outlook, Appl. Phys. Rev. 3 (2016) 011103.

[3]. T. F. Krauss, R. M. DelaRue, Photonic crystals in the optical regime - past, present and future, Prog. Quant. Electron. 23 (1999) 51-96.

[4]. V.A. Tolmachev, L.C. Hranicina, E.N. Vlasova, B.Z.Volchek, A.V.Naschekin, A.D.Remenyuk, E.V.Astrova, One-directional photonic crystal, obtained byvertical unizotropic etching of silicon, Phys.Tech.Semicond, 36 (2002) 996-1000 (in Russian).

[5]. M. Campbell, D. N. Sharp, M. T. Harrison, R. G. Denning, A. J. Turberfield, Fabrication of photonic crystals for the visible spectrum by holographic lithography, NATURE 404 (2000) 53-56.

[6]. G. P. Wang, C. Tan, Y. Yi, H. Shan, Holography for one-step fabrication of three-dimensional PC, J. of Modern Optics, 50 (2003) 2155-2161.

[7]. E. M. Arakcheeva, E. M.Tanklevskaja, S. I. Nesterov, M. V. Makcimov, S. A. Gurevich, J. Seekamp, C.M. Sotomayor Torres, Fabrication of photonic crystals by nanoimprint method in structures based on semiconductors and polymers, ZhTF, 75 (2005) 80-84 (in Russian).

[8]. D. George, J. Lutkenhaus, D. Lowell, M. Moazzezi, M. Adewole, U. Philipose, H. Zhang, Z. L. Poole, K. P. Chen, Y. Lin, Holographic fabrication of 3D photonic crystals through interference of multi-beams with $4+1,5+$ 1 and $6+1$ configurations, Optics Express 22 (2014) 22421-22431.

[9]. S. G. Romanov, A. V. Korovin, A. Regensburger, U. Peschel, Hybrid colloidal plasmonic-photonic crystals, Adv. Mat. 23 (2011) 2515-2533.

[10]. J. Burunkova , I. Denisiuk , N. Vorzobova , L. Daroczi , Cs. Hegedus , S. Charnovych, S. Kokenyesi, Fabrication and characterization of gold/acrylic polymer nanocomposites, Eur. Pol. J. 49 (2013) 3072-3077.

[11]. J. Burunkova, S. Kokenyesi, I. Csarnovics, A. Bonyár, M. Veres, A. Csík, Influence of gold nanoparticles on the photo-polymerization processes and structure in acrylate nanocomposites, European Polymer Journal 64 (2015) 189-195.

[12]. Y. Tomita, N. Suzuki, K. Chikama, Holographic manipulation of nanoparticle distribution morphology in nanoparticle-dispersed photopolymers, Opt. Lett. 30 (2005) 839-841.

[13]. M. A. Bruk, The Radiation Polymerisation of Monomers Adsorbed on Solid Surfaces, Russian Chemical Reviews 56 (1987) 81-96.

[14]. G. G. Suchkova, L. I. Maklakov. Amide bands in the IR spectra of urethanes, Vibrational Spectroscopy 51 (2009) 333-339. 
Figure 2
Click here to download high resolution image

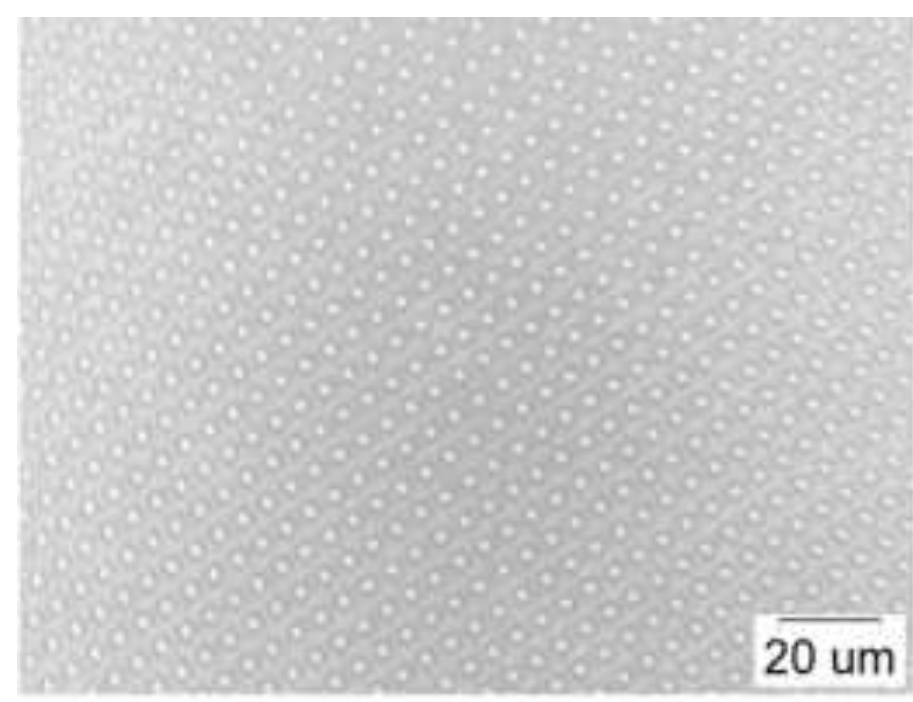

a

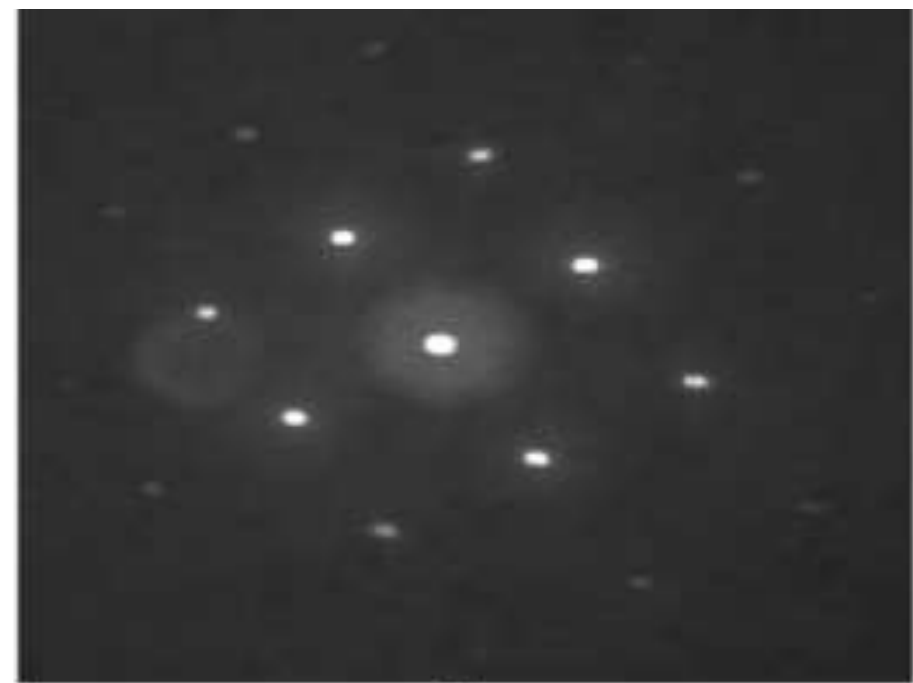

b

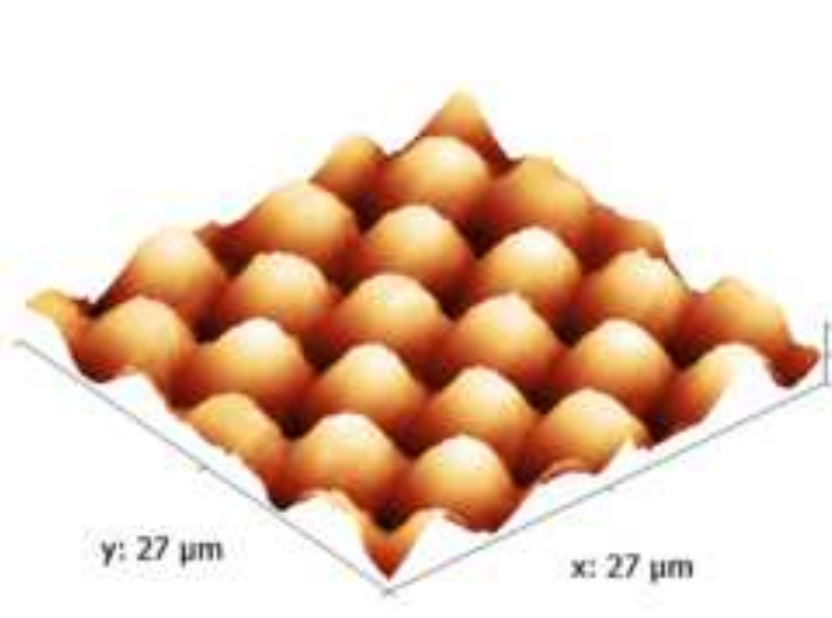

$\mathrm{c}$

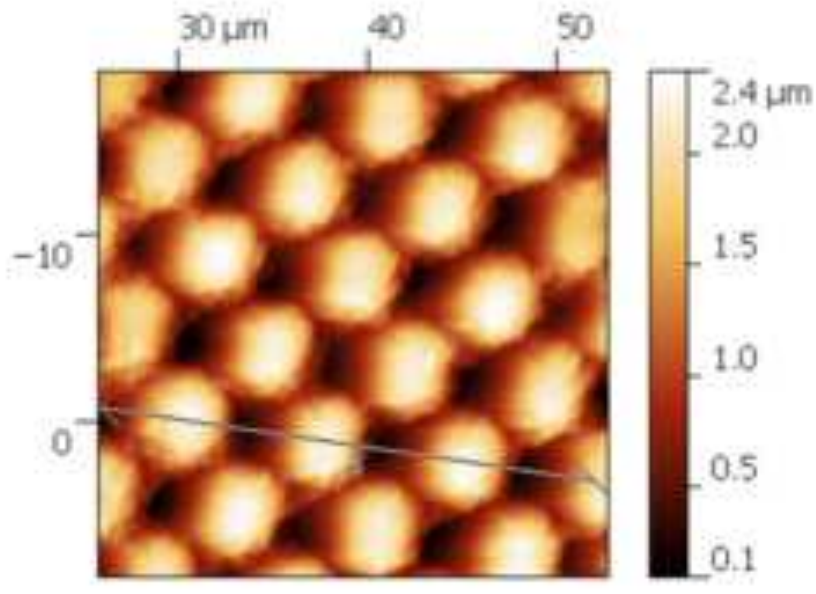

e
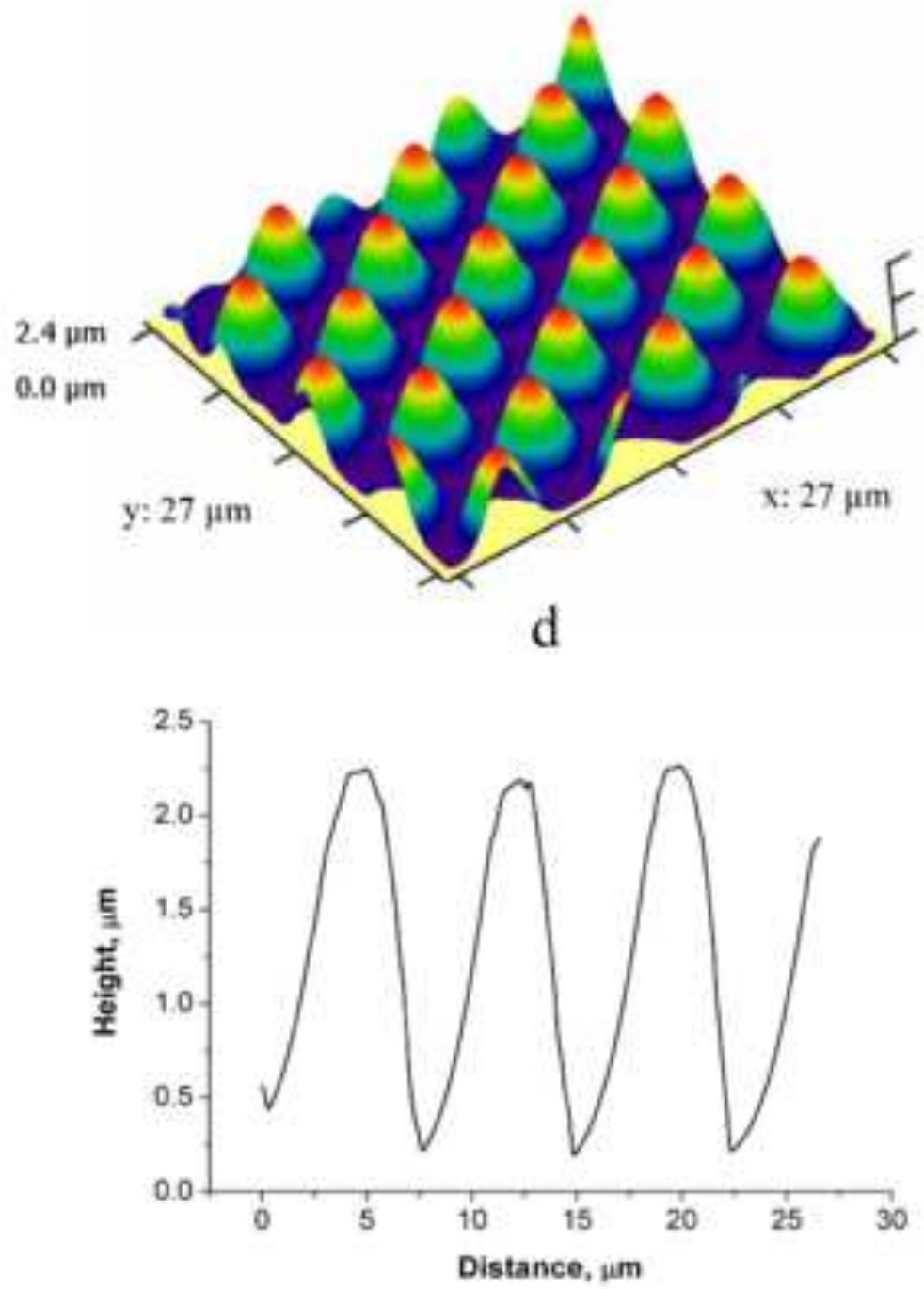

$\mathrm{f}$ 
Click here to download high resolution image

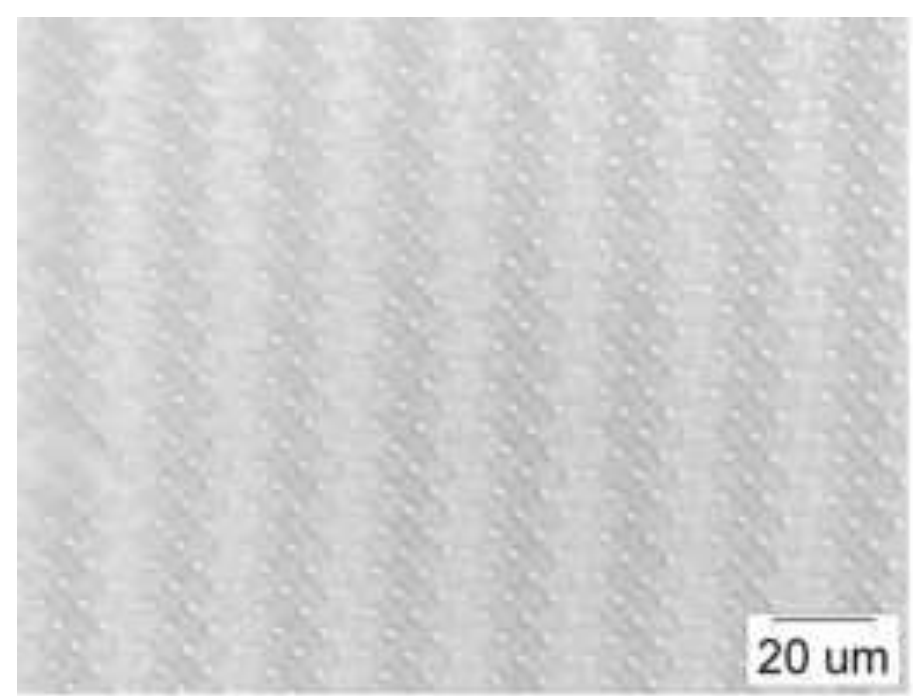

a

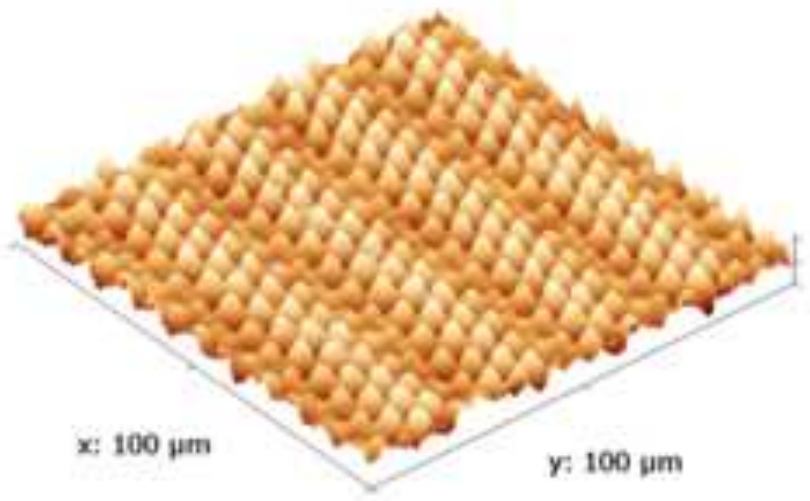

c

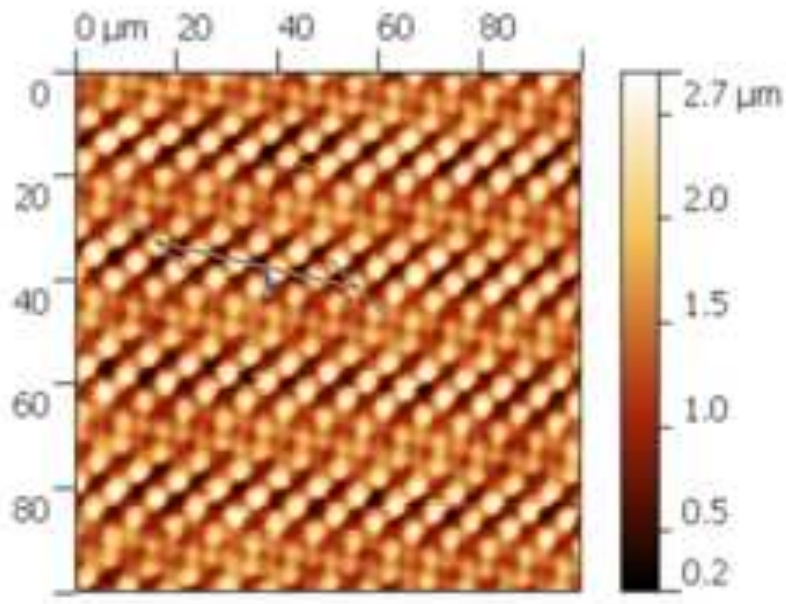

e

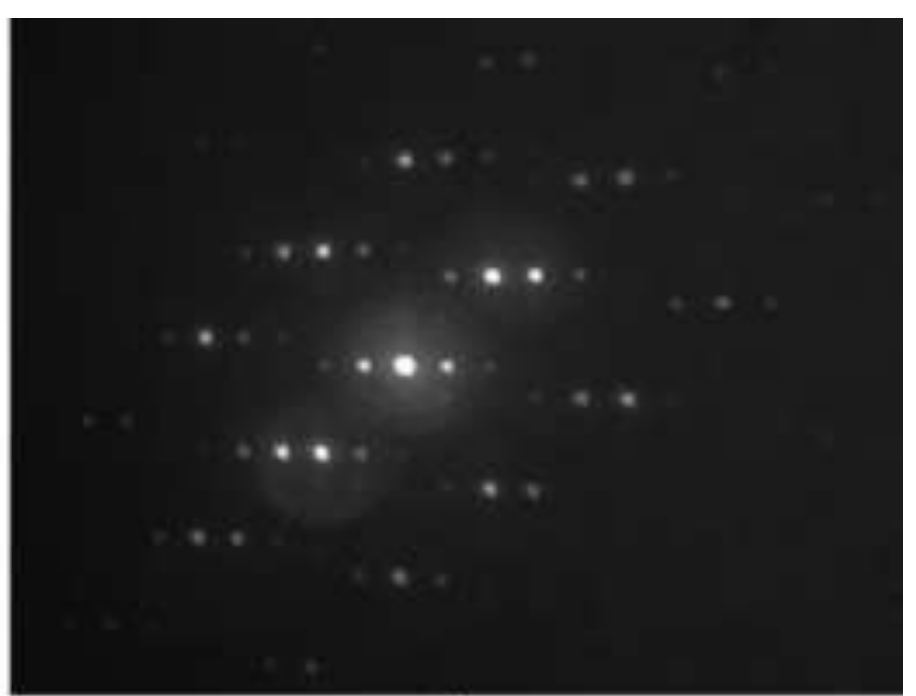

b

$2.7 \mathrm{pm}$ $0.0 \mu \mathrm{m}$

y: 100 นเก

x: 100 нат

d

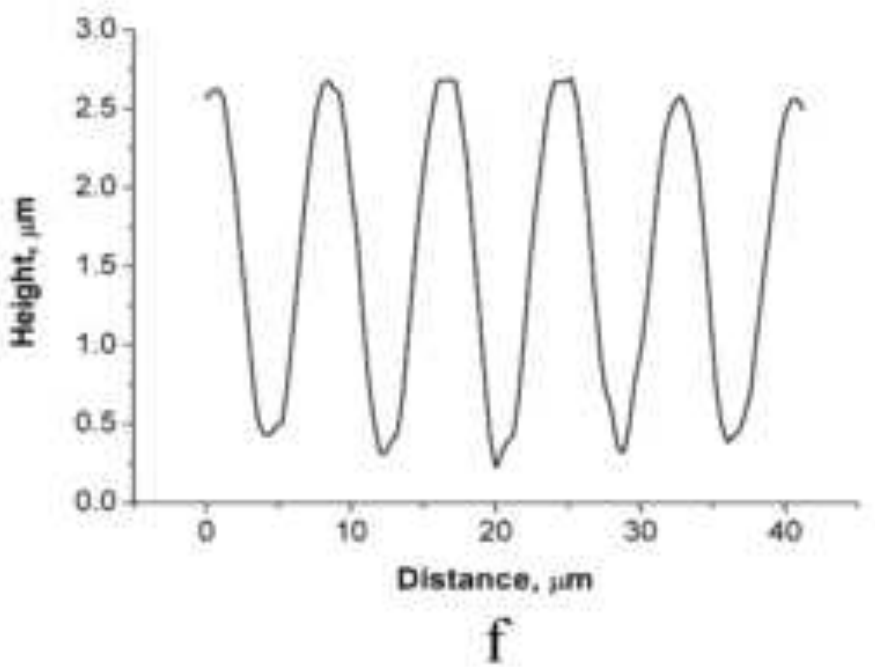




\section{Figure 4}

Click here to download high resolution image

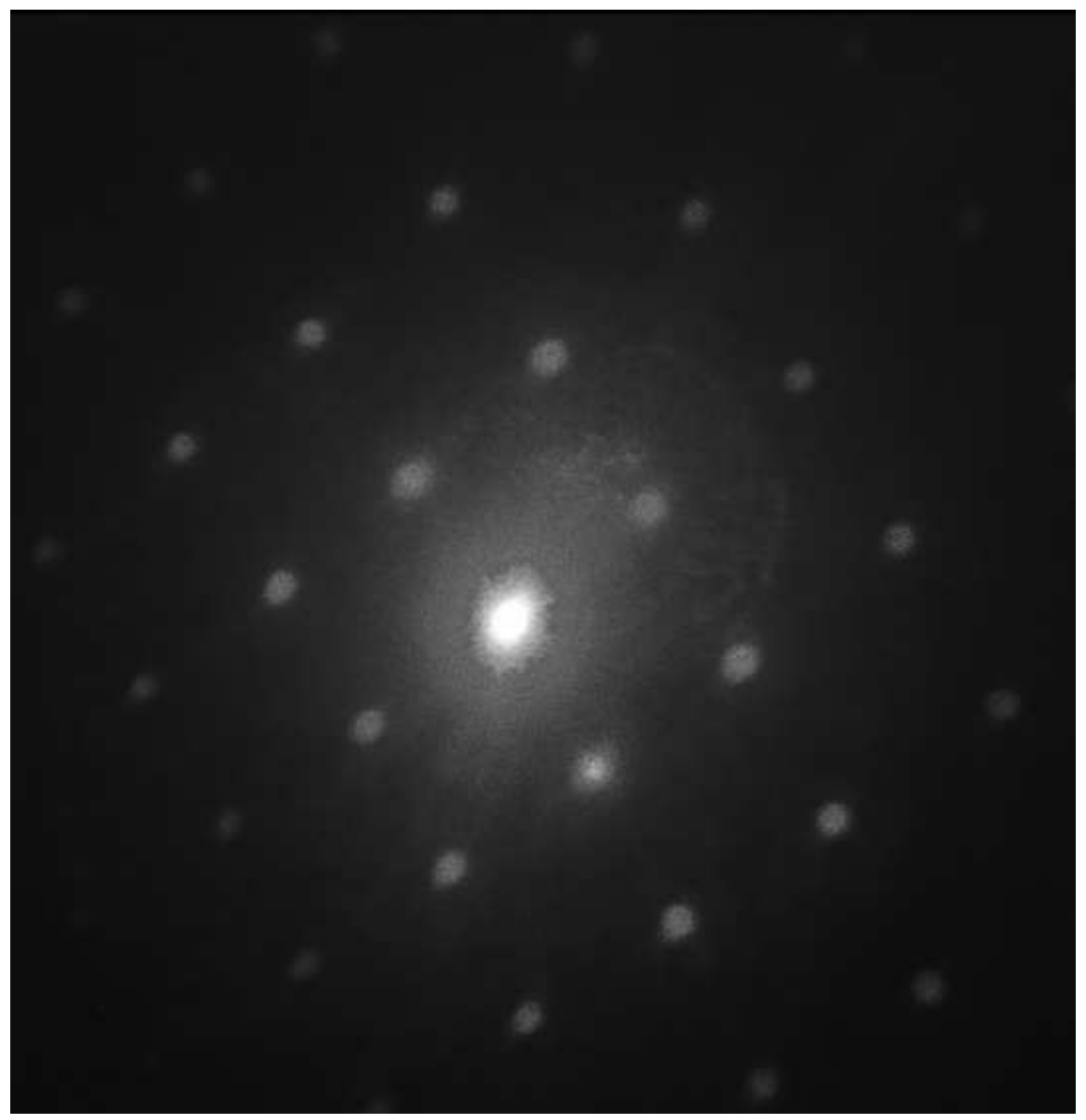




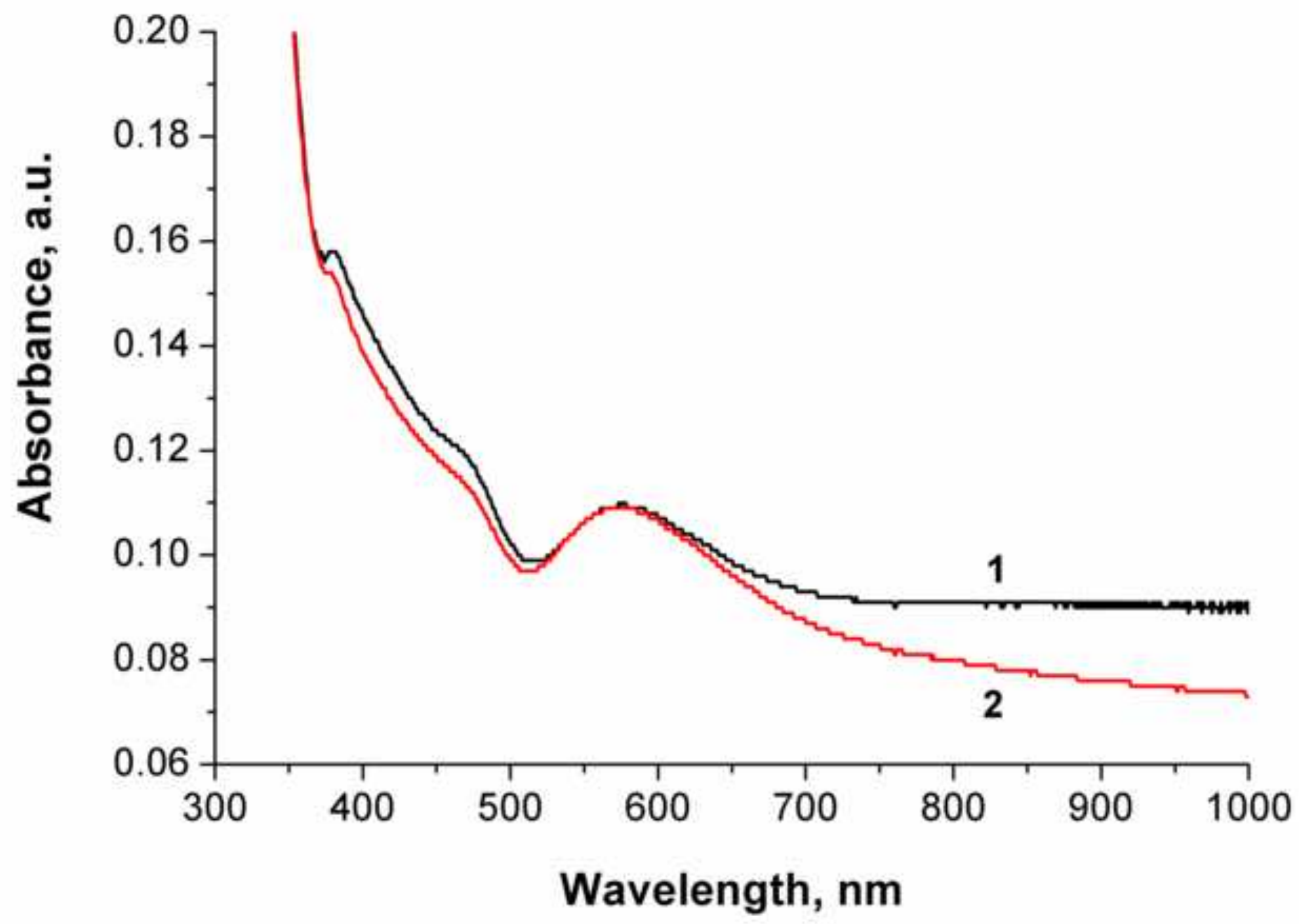



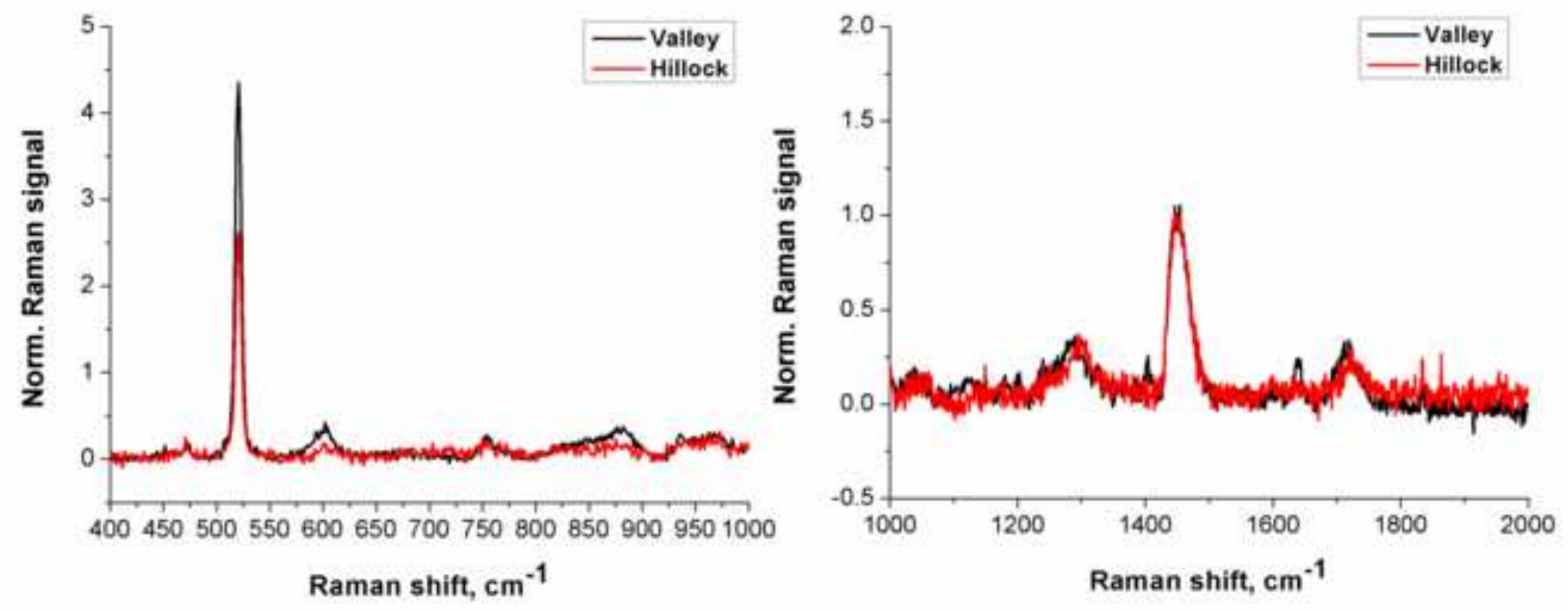


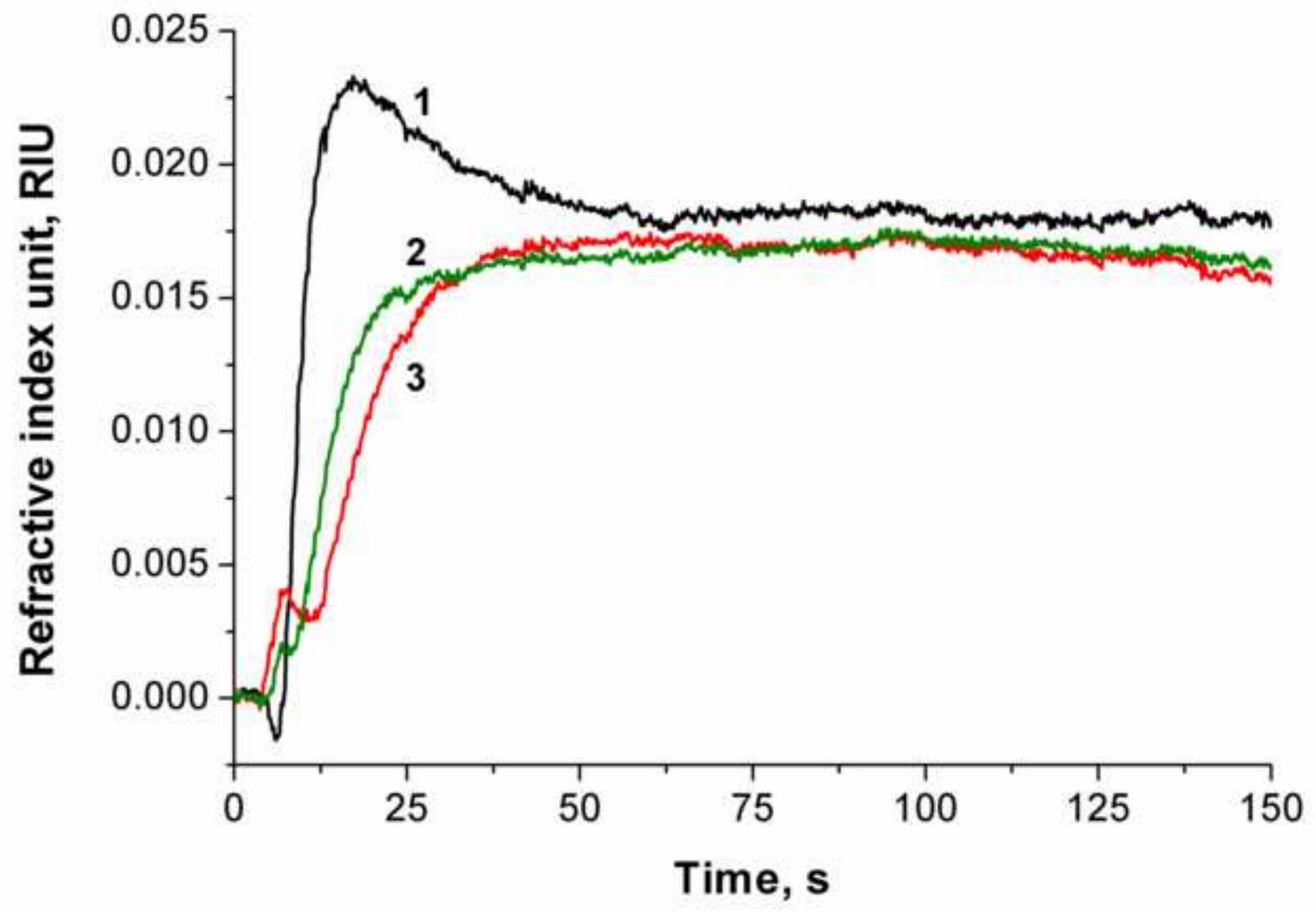



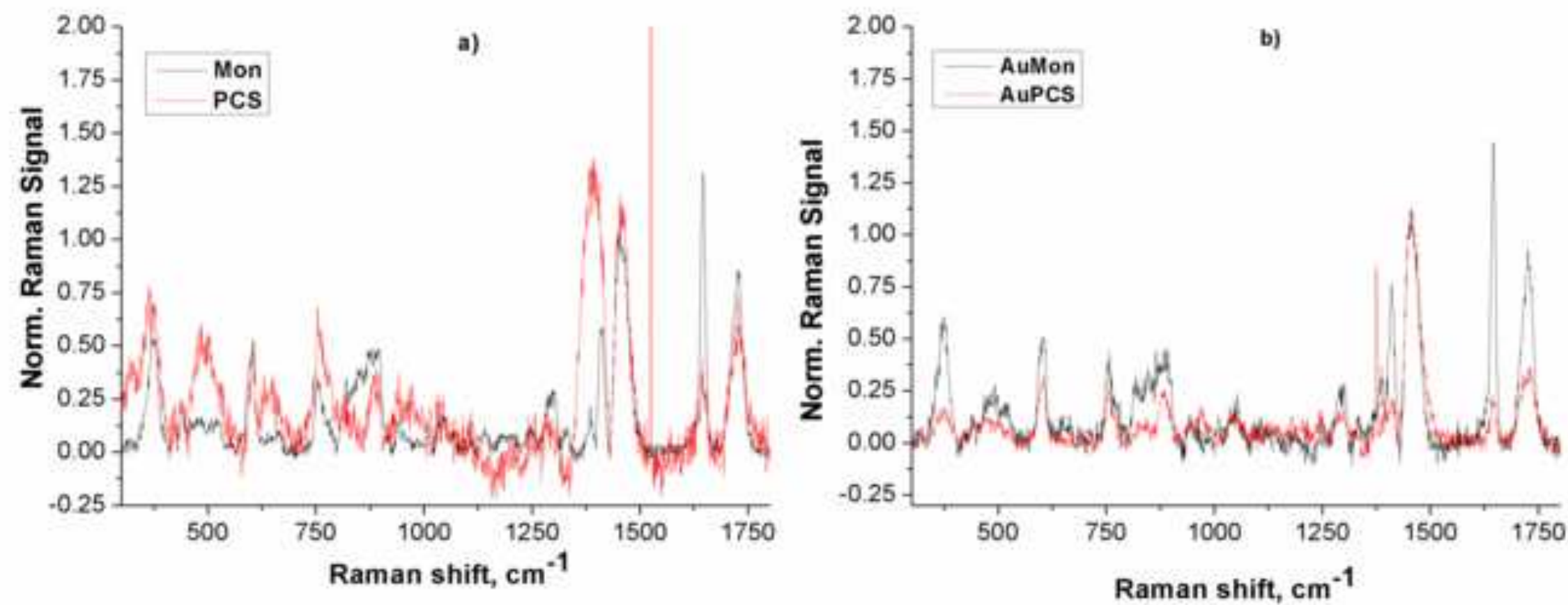

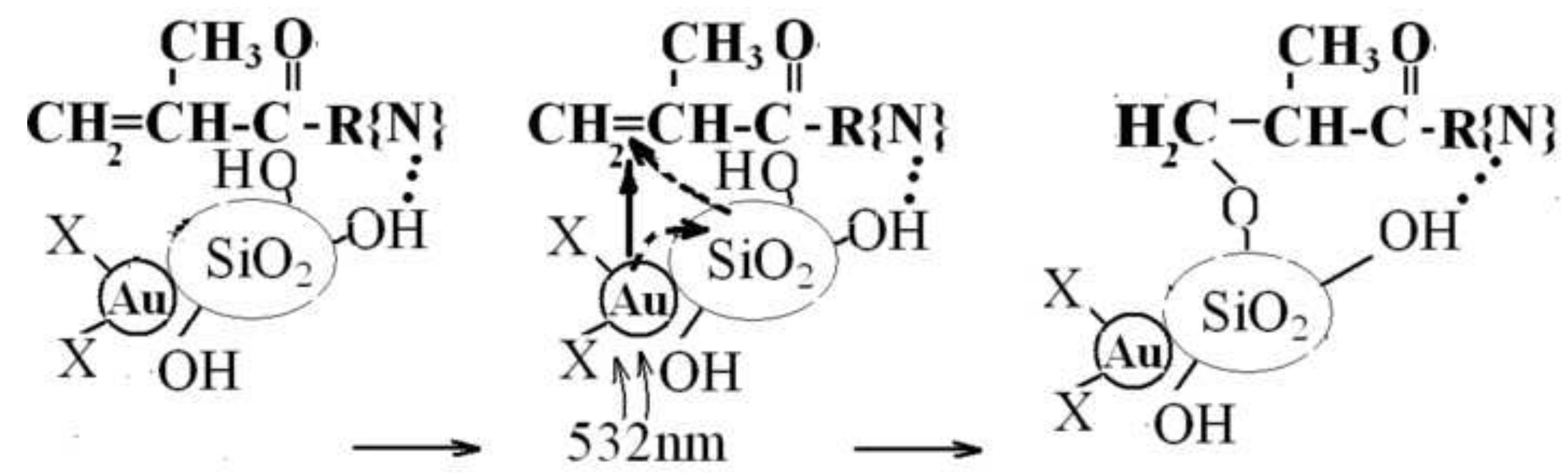